\title{
SN 2009E: a faint clone of SN 1987A
}

\author{
A. Pastorello ${ }^{1,2,3}$, M. L. Pumo ${ }^{1,4}$, H. Navasardyan ${ }^{1}$, L. Zampieri ${ }^{1}$, M. Turatto ${ }^{5}$, J. Sollerman ${ }^{6}$, \\ F. Taddia ${ }^{6}$, E. Kankare ${ }^{7}$, S. Mattila ${ }^{7}$, J. Nicolas ${ }^{8}$, E. Prosperi ${ }^{9}$, A. San Segundo Delgado ${ }^{10}$, S. Taubenberger ${ }^{11}$, \\ T. Boles ${ }^{12}$, M. Bachini ${ }^{13}$, S. Benetti ${ }^{1}$, F. Bufano ${ }^{4}$, E. Cappellaro ${ }^{1}$, A. D. Cason ${ }^{14}$, G. Cetrulo ${ }^{15}$, M. Ergon ${ }^{6}$, \\ L. Germany ${ }^{16}$, A. Harutyunyan ${ }^{17}$, S. Howerton ${ }^{18}$, G. M. Hurst ${ }^{19}$, F. Patat ${ }^{20}$, M. Stritzinger ${ }^{6,21}$, \\ L.-G. Strolger ${ }^{22}$, and W. Wells ${ }^{23}$ \\ (Affiliations can be found after the references)
}

Received 16 September 2011 / Accepted 3 November 2011

\begin{abstract}
Context. 1987A-like events form a rare sub-group of hydrogen-rich core-collapse supernovae that are thought to originate from the explosion of blue supergiant stars. Although SN 1987A is the best known supernova, very few objects of this group have been discovered and, hence, studied. Aims. In this paper we investigate the properties of SN 2009E, which exploded in a relatively nearby spiral galaxy (NGC 4141) and that is probably the faintest 1987A-like supernova discovered so far. We also attempt to characterize this subgroup of core-collapse supernovae with the help of the literature and present new data for a few additional objects.

Methods. The lack of early-time observations from professional telescopes is compensated by frequent follow-up observations performed by a number of amateur astronomers. This allows us to reconstruct a well-sampled light curve for SN 2009E. Spectroscopic observations which started about 2 months after the supernova explosion, highlight significant differences between SN 2009E and the prototypical SN 1987A. Modelling the data of SN 2009E allows us to constrain the explosion parameters and the properties of the progenitor star, and compare the inferred estimates with those available for the similar SNe 1987A and 1998A.

Results. The light curve of SN 2009E is less luminous than that of SN 1987A and the other members of this class, and the maximum light curve peak is reached at a slightly later epoch than in SN 1987A. Late-time photometric observations suggest that SN 2009E ejected about $0.04 M_{\odot}$ of ${ }^{56} \mathrm{Ni}$, which is the smallest ${ }^{56} \mathrm{Ni}$ mass in our sample of 1987A-like events. Modelling the observations with a radiation hydrodynamics code, we infer for SN 2009E a kinetic plus thermal energy of about 0.6 foe, an initial radius of $\sim 7 \times 10^{12} \mathrm{~cm}$ and an ejected mass of $\sim 19 M_{\odot}$. The photospheric spectra show a number of narrow $\left(v \approx 1800 \mathrm{~km} \mathrm{~s}^{-1}\right)$ metal lines, with unusually strong Ba II lines. The nebular spectrum displays narrow emission lines of $\mathrm{H}, \mathrm{Na} \mathrm{I},[\mathrm{Ca} \mathrm{II}]$ and $[\mathrm{O} \mathrm{I}]$, with the [O I] feature being relatively strong compared to the [Ca II] doublet. The overall spectroscopic evolution is reminiscent of that of the faint ${ }^{56} \mathrm{Ni}$-poor type II-plateau supernovae. This suggests that SN $2009 \mathrm{E}$ belongs to the low-luminosity, low ${ }^{56} \mathrm{Ni}$ mass, low-energy tail in the distribution of the 1987A-like objects in the same manner as SN 1997D and similar events represent the faint tail in the distribution of physical properties for normal type II-plateau supernovae.
\end{abstract}

Key words. supernovae: general - supernovae: individual: SN 2009E - supernovae: individual: SN 1987A - supernovae: individual: SN 1998A

\section{Introduction}

The explosion of supernova (SN) 1987A in the Large Magellanic Cloud (LMC) was an epic event not only because it was the nearest $\mathrm{SN}$ in a period of about 4 centuries, but also because it changed significantly the general understanding of the destiny of massive stars. It was commonly believed that hydrogen-rich (H-rich) type II plateau supernovae (SNe IIP) were generated by the explosion of red supergiant (RSG) stars. However, the unusual photometric evolution of SN 1987A, with a broad light curve peak reached about 3 months after the explosion instead of the classical plateau observed in $\mathrm{H}$-rich $\mathrm{SNe}$, and the direct detection of the progenitor star in pre-explosion images changed this general view and proved that the precursor of this $\mathrm{SN}$ in the LMC was instead a blue supergiant (BSG, Arnett et al. 1989, and references therein).

Due to their intrinsic rarity and faint early time luminosity, only very few 1987A-like objects were discovered in the

\footnotetext{
* Appendices A and B are available in electronic form at http://www . aanda.org

$\star \star$ Full Table 2 is only available in electronic form at the CDS via anonymous ftp to cdsarc.u-strasbg.fr (130.79.128.5) or via http://cdsarc.u-strasbg.fr/viz-bin/qcat?J/A+A/537/A141
}

past. Good datasets therefore exist only for a handful of objects (SN 1998A, Woodings et al. 1998; Pastorello et al. 2005, SN 2000cb, Hamuy 2001; Kleiser et al. 2011, SNe 2006V and 2006au, Taddia et al. 2011). In analogy with SN 1987A, some of these objects show broad, delayed light curve peaks in all bands (e.g. SN 1998A), while others (e.g. SN 2000cb and SN 1982F) show1987A-likeness only in the red bands, while the blue bands display a rather normal type IIP behaviour.

In this context, the discovery of a new sub-luminous $\mathrm{SN}$ with a light curve comparable to that of SN 1987A, but with other observed properties (fainter intrinsic luminosity at peak, smaller synthesized ${ }^{56} \mathrm{Ni}$ mass and lower expansion velocity of the ejected material) resembling those of some underluminous type IIP SNe (Turatto et al. 1998; Benetti et al. 2001; Pastorello et al. 2004, 2006, 2009) is even more interesting.

This paper is organized as follows: in Sect. 2 we give basic information on SN 2009E and its host galaxy, and we present photometric and spectroscopic observations of the SN. In Sect. 3 we discuss the properties of the progenitor star and the explosion parameters as derived from the characteristics of the nebular spectrum and by modelling the SN observations. In Sect. 4 we analyse the general properties of the family of $\mathrm{SNe}$ similar to 
SN 1987A, whilst the rate of these events is computed in Sect. 5. Main conclusions are given in Sect. 6. Finally, an Appendix has been included to present our sample of 1987A-like events (Appendix A), and to compare the light curves of well monitored objects with those of SN 1987A (Appendix B).

\section{Observations of SN 2009E}

\subsection{The host galaxy}

NGC 4141 is classified by HyperLeda ${ }^{1}$ as an SBc galaxy, rich in $\mathrm{H}$ II regions $\left(\mathrm{NED}^{2}\right)$. Kewley et al. (2005) estimated the integrated oxygen abundance to be $\log (\mathrm{O} / \mathrm{H})+12=8.60(8.74$ in the nucleus). They also estimated a star formation rate (SFR) from the integrated flux of $\mathrm{H} \alpha$, viz. $\sim 0.6 M_{\odot} \mathrm{yr}^{-1}$.

Adopting the recessional velocity corrected for Local Group infall into Virgo quoted by HyperLeda $\left(v_{\text {Vir }}=2158 \pm 20 \mathrm{~km} \mathrm{~s}^{-1}\right)$ and a Hubble constant $H_{0}=72 \pm 5 \mathrm{~km} \mathrm{~s}^{-1} \mathrm{Mpc}^{-1}$, we obtain a distance of about $29.97 \pm 2.10 \mathrm{Mpc}$ (i.e. distance modulus $\mu=32.38 \pm 0.35 \mathrm{mag})$. The Galactic extinction in the direction of NGC 4141 is very low, i.e. $E(B-V)=0.02$ mag (Schlegel et al. 1998). Narrow interstellar $\mathrm{Na} I \lambda \lambda 5889,5895$ (hereafter $\mathrm{Na}$ ID) absorption at the host galaxy rest wavelength is marginally detected in the SN spectra, with an equivalent width (EW) of about $0.12 \AA$. Adopting the relation between $\mathrm{EW}_{\mathrm{NaID}}$ and interstellar extinction from Turatto et al. (2003), we find a host galaxy reddening of $E(B-V)=0.02 \mathrm{mag}^{3}$. The total colour excess in the direction of SN 2009E is therefore $E(B-V)=0.04$ mag.

\subsection{The discovery of SN $2009 E$}

SN 2009E was discovered in the spiral galaxy NGC 4141 on January 3rd, 2009, at an unfiltered magnitude of 17.8 (Boles 2009). With the distance and reddening estimated in Sect. 2.1, the absolute magnitude at the discovery was about -14.7 . The position of $\mathrm{SN} 2009 \mathrm{E}$ is $\alpha=12^{\mathrm{h}} 09^{\mathrm{m}} 49.56 \pm 0.03, \delta=$ $+58^{\circ} 50^{\prime} 50^{\prime} \cdot 3 \pm 00^{\prime} 1$ (equinox $\mathrm{J} 2000.0$ ), which is $17^{\prime \prime} .2$ East and 6.' 9 South of the center of the host galaxy. According to Boles (2009), nothing was visible in pre-explosion images obtained on 2008 February 6th (to limiting magnitude of 19.5). We also found pre-explosion archive $g^{\prime}, R_{\mathrm{c}}$ and $I_{\mathrm{c}}$ images ${ }^{4}$ obtained on 2008 February 24th at the $0.5-\mathrm{m}$ telescope of the Akeno Observatory/ICRR (Yamanashi, Japan) and no source brighter than $V=19.5, R=19.6, I=18.8$ mag was visible at the $\mathrm{SN}$ position. In this paper, we will adopt January 1.0 UT $(\mathrm{JD}=2454832.5$, see Sect. 2.3) as an indicative epoch for the core-collapse.

NGC 4141 also hosted the type II SN 2008X (Boles 2008; Madison et al. 2008; Blondin et al. 2008), that exploded 7"'6 east and 4.' 6 north of the nucleus of the galaxy. Interestingly, SN 2008X was another sub-luminous $\left(M_{R} \approx-14.9\right.$, Boles 2008) event. The classification spectrum of SN 2008X obtained a few weeks after the explosion showed that it was a type II SN (Blondin et al. 2008), although with rather narrow spectral lines, similar to those observed in SN 2005cs (Pastorello et al. 2006).

Probably the faint apparent magnitude of SN 2009E discouraged astronomers to promptly classify this object. However, later

\footnotetext{
1 http://leda.univ-lyon $1 . \mathrm{fr} /$

2 http://nedwww.ipac. caltech.edu/index.html

3 Note, however, that Poznanski et al. (2011) casted doubt on the robustness of the correlation between EW (Na ID) and interstellar extinction as determined from low resolution type Ia SN spectra.

4 Images have been downloaded through the SMOKA Data Archive (Baba et al. 2002).
}

Table 1. Magnitudes of reference stars in the SN field, calibrated on several photometric nights.

\begin{tabular}{lccccc}
\hline \hline Star & $U$ & $B$ & $V$ & $R$ & $I$ \\
\hline 1 & $18.86(0.03)$ & $17.59(0.02)$ & $16.52(0.02)$ & $15.74(0.01)$ & $15.18(0.01)$ \\
2 & $17.47(0.02)$ & $16.88(0.01)$ & $16.11(0.01)$ & $15.60(0.01)$ & $15.20(0.01)$ \\
3 & $15.85(0.04)$ & $15.87(0.01)$ & $15.35(0.01)$ & $15.01(0.01)$ & $14.69(0.01)$ \\
4 & $18.07(0.03)$ & $16.89(0.01)$ & $15.41(0.02)$ & - & - \\
5 & $18.81(0.03)$ & $18.73(0.01)$ & $18.05(0.02)$ & $17.62(0.01)$ & $17.19(0.02)$ \\
6 & $17.75(0.03)$ & $17.85(0.01)$ & $17.26(0.01)$ & $16.83(0.01)$ & $16.43(0.01)$ \\
7 & $20.76(0.05)$ & $19.33(0.02)$ & $18.17(0.01)$ & $17.31(0.01)$ & $16.56(0.02)$ \\
8 & $18.97(0.05)$ & $18.91(0.02)$ & $18.23(0.01)$ & $17.78(0.01)$ & $17.37(0.01)$ \\
9 & - & $19.90(0.02)$ & $18.46(0.02)$ & $17.47(0.01)$ & $16.58(0.02)$ \\
10 & - & $16.32(0.01)$ & $15.70(0.02)$ & $15.31(0.02)$ & $14.94(0.01)$ \\
11 & - & $14.45(0.02)$ & $13.73(0.03)$ & $13.15(0.04)$ & $12.83(0.01)$ \\
12 & - & $16.43(0.03)$ & $15.86(0.03)$ & $15.48(0.01)$ & $15.11(0.02)$ \\
13 & $16.63(0.06)$ & $16.40(0.01)$ & $15.67(0.02)$ & $15.22(0.01)$ & $14.78(0.02)$ \\
14 & - & $19.86(0.06)$ & $18.27(0.10)$ & - & - \\
\hline
\end{tabular}

Notes. The root mean square of the average magnitudes are reported in brackets.

on, Prosperi \& Hurst (2009) noted that SN 2009E brightened by $1-1.5$ mag during the subsequent two months, and only at about 80 days from the discovery the object was classified by Navasardyan et al. (2009) as a type II SN around the end of the $\mathrm{H}$ recombination phase. SN 2009E appeared to show some similarity with the peculiar SN 1987A, especially in the late-time light curve brightening and the unusual strength of the Ba II P-Cygni lines. The most obvious differences were in the lower luminosity and the much narrower spectral features.

\subsection{Light curves}

Whilst professional astronomers initially missed to follow this transient object, amateur astronomers monitored SN 2009E extensively, and it is thanks to their efforts that we can now recover information on the early flux evolution. Most images collected during the period January 2009 to May 2009 were from amateur observations. Since the end of March, we started multiband photometric and spectroscopic follow-up observations with larger, professional telescopes. All photometric data were reduced with standard techniques in IRAF $^{5}$. Images were first overscan, bias and flat-field corrected. Then, photometric measurements of filtered images were performed using a PSF-fitting technique because suitable template images were not available. However, since the SN location was quite peripheral in the galaxy arm, its luminosity largely exceeded that of the surroundings, except at very late epochs when the $\mathrm{SN}$ became weak. We estimated the uncertainty due to the non-flat background by placing in all images several artificial stars close to the $\mathrm{SN}$ location. The adopted errors were the rms of the recovered artificial star PSF magnitudes. Multi-band zero-points for the different nights were computed through observations of standard stellar fields (Landolt 1992) obtained during the same nights as the SN observations. The SN magnitudes were finally fine tuned with reference to a sequence of stars in the field of NGC 4141 (see Fig. 1), calibrated by averaging magnitudes measured in selected photometric nights. The magnitudes of the local sequence stars are shown in Table 1, while the final SN magnitudes are reported in Table 2.

5 IRAF is distributed by the National Optical Astronomy Observatories, which are operated by the Association of Universities for Research in Astronomy, Inc., under cooperative agreement with the National Science Foundation. 
Table 2. A small sample of our calibrated $U, B, V, R, I$ band magnitudes of SN 2009E.

\begin{tabular}{cccccccc}
\hline \hline Date & $J D$ & $U$ & $B$ & $V$ & $R$ & $I$ & Instrument \\
\hline Feb. 06 2008 & 2454502.5 & - & - & - & $>19.5$ & - & $1 \star$ \\
Feb. 24 2008 & 2454521.22 & - & - & $>19.5 \ddagger$ & $>19.6$ & $>18.9$ & 2 \\
Jan. 03 2009 & 2454834.56 & - & - & - & $17.79(0.08)$ & - & $1 \star$ \\
Jan. 03 2009 & 2454835.42 & - & - & - & $18.01(0.15)$ & - & $1 \star$ \\
Jan. 05 2009 & 2454836.56 & - & - & - & $17.87(0.10)$ & - & $3 \star$ \\
Jan. 05 2009 & 2454836.71 & - & - & - & $17.86(0.25)$ & - & $4 \star$ \\
Jan. 07 2009 & 2454838.51 & - & - & - & $17.88(0.12)$ & - & $5 \star$ \\
Jan. 09 2009 & 2454840.84 & - & - & $18.04(0.33)$ & $17.71(0.21)$ & - & 6 \\
Jan. 13 2009 & 2454844.63 & - & - & - & $17.57(0.12)$ & - & $4 \star$ \\
Jan. 16 2009 & 2454848.44 & - & - & - & $17.42(0.19)$ & - & $6 \star$ \\
Jan. 18 2009 & 2454849.80 & - & - & - & $17.40(0.12)$ & - & $7 \star$ \\
Jan. 26 2009 & 2454858.01 & $>20.35$ & $18.91(0.29)$ & $17.66(0.13)$ & $17.28(0.11)$ & $16.93(0.07)$ & 8 \\
\hline
\end{tabular}

Notes. The full photometry is available in electronic form at the CDS. The numbers in column 8 identify the different instrumental configurations. Unfiltered observations rescaled to $R$-band magnitudes are marked with the symbol “ $\star$ ”. The symbol “ $\ddagger$ ” marks a $g$ '-band observation converted into Johnson-Bessell $V$ band. $1=0.36-\mathrm{m} \mathrm{C14}$ reflector + Apogee AP7 CCD camera (Obs. T. Boles, Coddendham Observatory, Suffolk, UK); 2. 0.50-m Telescope + Apogee U6 CCD camera (Obs. M. Yoshida, Akeno Observatory/ICRR, Yamanashi, Japan); 3. 0.28-m C11 reflector + SBIG ST-8XME Kaf1602E CCD camera (Obs. J. Nicolas, Vallauris, France); 4. 0.28-m C11 reflector + SBIG ST-8XME Kaf1602E CCD camera (Obs. J. M. Llapasset, Perpignan, France); 5. 0.36-m C14 reflector + Apogee AP7 CCD camera (Obs. O. Trondal, Groruddalen, Oslo, Norway); 6. 0.36-m Meade LX200 Telescope + SBIG ST-9XE CCD camera (Obs. E. Prosperi, Osservatorio Astron. di Castelmartini, Larciano, Italy); 7. 0.3-m Takahashi Mewlon 300 + SBIG ST-8E NABG camera (obs. W. Wells, Gras-002, New Mexico, USA); 8. 2.0-m Faulkes Telescope North + EM01 (Faulkees Telescope Archive - Las Cumbres Observatory, Mt. Haleakala, Hawaii Islands, USA).

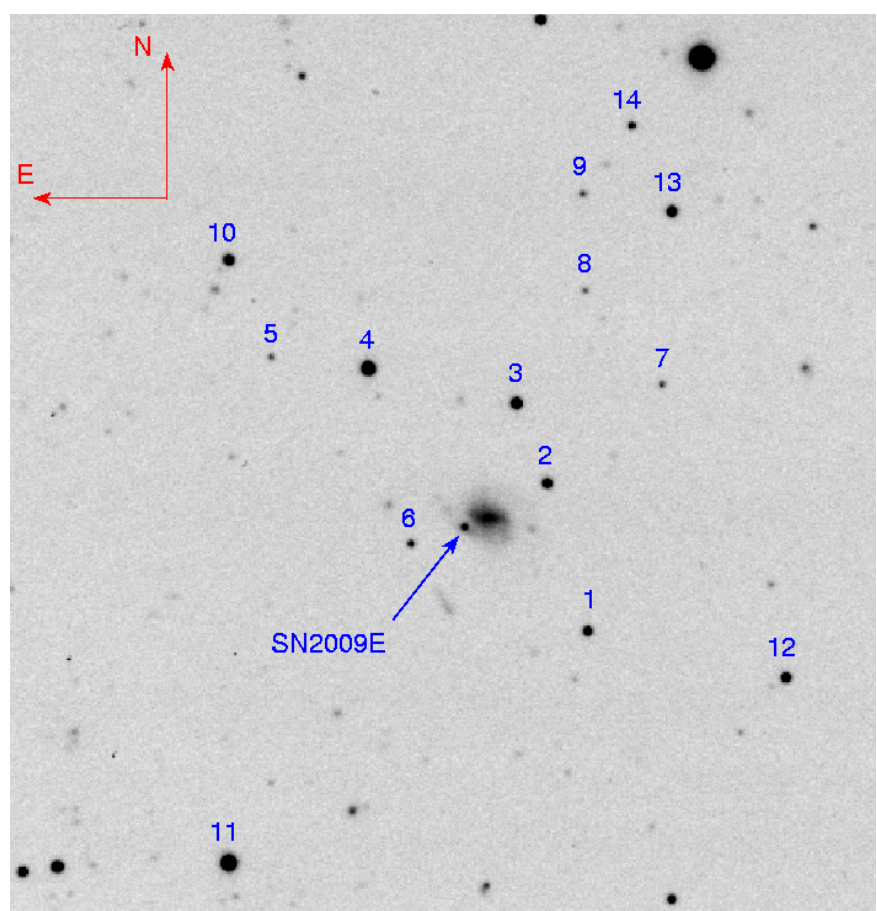

Fig. 1. SN 2009E in NGC 4141. Unfiltered image obtained on April 29, 2009 by J. N. with a $0.28-\mathrm{m} f / 6.5$ reflector equipped with a ST8XME with Kaf1602E CCD. Our sequence of reference stars is marked by numbers. The field of view is about $10^{\prime} \times 10^{\prime}$.

Since a number of unfiltered pre-explosion images of NGC 4141 were available, magnitude measurements on the amateurs' images were performed with the PSF-fitting technique after subtraction of the best seeing host galaxy template image. In this way we minimized the contamination of the background sources near the SN location. The subsequent photometric calibration was performed with the prescriptions discussed in Pastorello et al. (2008). Since the detectors used by amateur astronomers in their follow-up campaign of SN 2009E have quantum efficiencies preferentially peaking at 5700-6500 A,

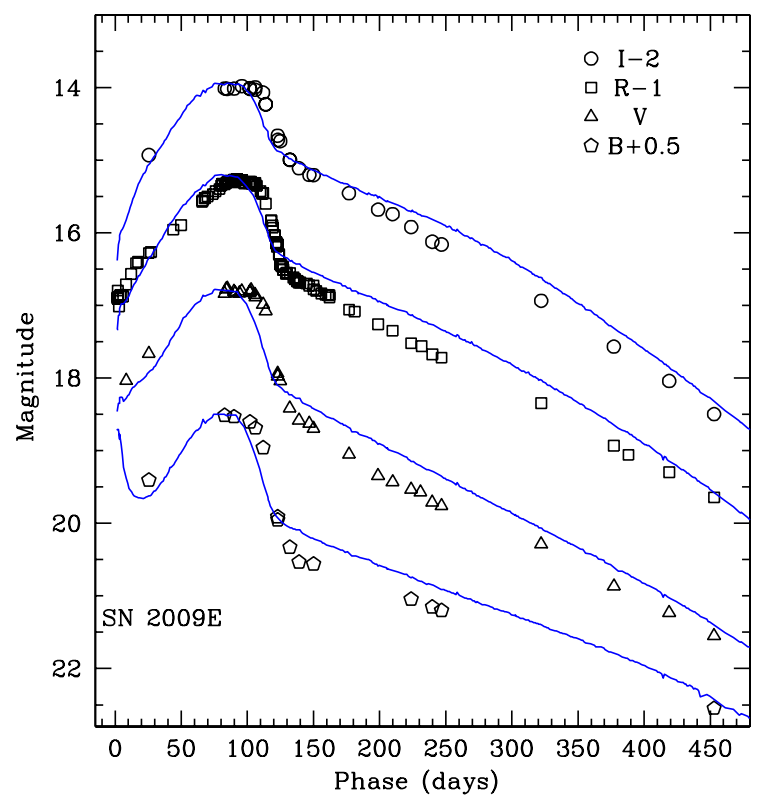

Fig. 2. $B, V, R$ and $I$-band light curves of $\mathrm{SN} 2009 \mathrm{E}$ compared with those of SN 1987A (solid blue line; Menzies et al. 1987; Catchpole et al. 1987, 1988, 1989; Whitelock et al. 1988, 1989), shifted arbitrarily in magnitude to match the peak magnitudes of SN 2009E.

unfiltered magnitudes were scaled to match the $R$-band photometry. The SN magnitude errors accounted for the uncertainties in this convertion. The final $R$-band-calibrated magnitudes are reported in Table 2, marked with an asterisk. The $B, V, R, I$ light curves of SN 2009E, compared with those of SN 1987A, are shown in Fig. 2.

In Fig. 3 we compare the $B-V$ and $V-I$ colour curves of SN 2009E with those of a subsample of 1987A-like events and with the sub-luminous type IIP SN $2005 \mathrm{cs}$. The colour curves of SN 2009E are remarkably similar to those of SN 1987A at all phases. The colour estimates at phase $\sim 25$ days $(B-V \approx 1.2$ and $V-I \approx 0.7 \mathrm{mag}$ ) suggest that, in analogy with SN 1987A, $\mathrm{SN} 2009 \mathrm{E}$ became red in a time scale that is shorter than 
Table 3. Spectroscopic observations of SN 2009E.

\begin{tabular}{lcccccc}
\hline \hline Date & Average JD & Phase $^{\star}$ & Instrumental configuration & Exposure $(\mathrm{s})$ & Resolution $(\AA)$ & Range $(\AA)$ \\
\hline Mar. 24, 2009 & 2454915.40 & +82.9 & Ekar1.82m+AFOSC+gr.4 & 3600 & 34 & $3490-7790$ \\
Mar. 25, 2009 & 2454915.54 & +83.0 & Asiago1.22m+B\&C+g600 & 3600 & 6.1 & $5090-7500$ \\
Apr. 11, 2009 & 2454922.52 & +90.0 & NOT+ALFOSC+gr4 & 1200 & 13 & $3360-9100$ \\
Apr. 12, 2009 & 2454934.48 & +102.0 & NOT+ALFOSC+gr4 & 1200 & 179 & $3230-9060$ \\
Apr. 16, 2009 & 2454938.42 & +105.9 & CAHA2.2m+CAFOS+g200 & 1792 & 9.5 & $3700-10670$ \\
May 04, 2009 & 2454955.57 & +123.1 & NOT+ALFOSC+gr4 & 3700 & 13 & $3350-9120$ \\
May 13, 2009 & 2454964.62 & +132.1 & TNG+LRS+LRR & 3600 & 12 & $5050-9520$ \\
May 20, 2009 & 2454971.55 & +139.1 & TNG+LRS+LRR & 3600 & 12 & $4960-9560$ \\
Jun. 26, 2009 & 2455009.42 & +176.9 & CAHA2.2m+CAFOS+g200 & $2 \times 2400$ & 9.5 & $4100-9100$ \\
Jul. 18, 2009 & 2455031.44 & +198.9 & CAHA2.2m+CAFOS+g200+GG495 & $2 \times 3600$ & 13 & $4800-9690$ \\
Aug. 15, 2009 $\dagger$ & 2455058.84 & +226.3 & CAHA2.2m+CAFOS+g200 & $2 \times 3600$ & 12 & $3880-10670$ \\
Jan. 22, 2010 & 2455218.64 & +386.1 & NOT+ALFOSC+gr5 & $3 \times 1200$ & 14 & $5000-10000$ \\
Jan. 24, 2010 & 2455220.70 & +388.2 & NOT+ALFOSC+gr4 & $3 \times 1200$ & 12 & $3550-9080$ \\
\hline
\end{tabular}

Notes. ${ }^{\star}$ Days from explosion $(\mathrm{JD}=2454832.5) ; \bullet$ As estimated from the full width at half maximum of isolated night sky lines. ${ }^{\star}$ Poor signal-to-noise spectrum; ${ }^{\dagger}$ Weighted average of 2 spectra obtained on August 14 th and 15 th.

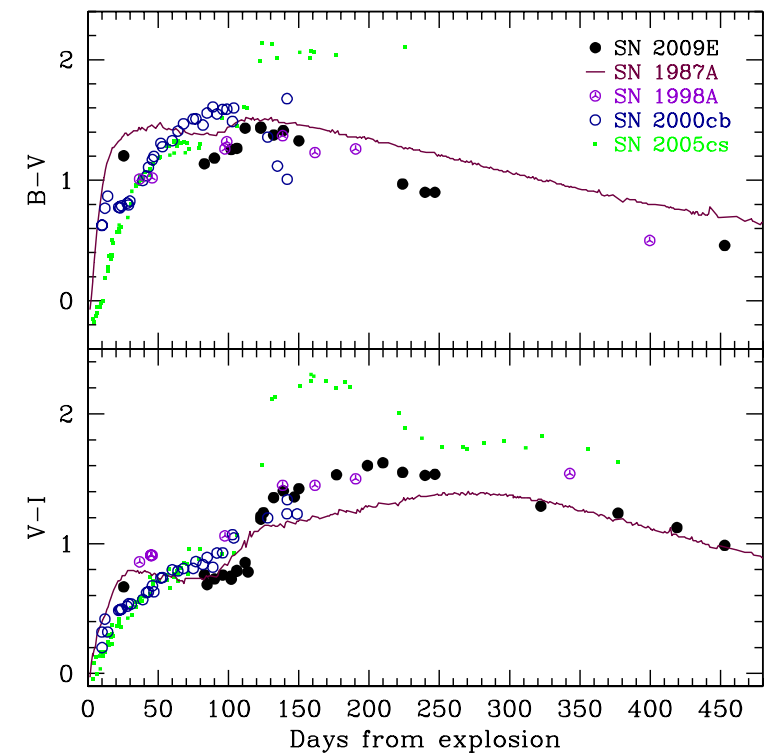

Fig. 3. $B-V($ top $)$ and $V-I$ (bottom) colour curves of SN 2009E compared with those of a subsample of 1987A-like events with multi-colour photometric coverage: SNe 1987A (Menzies et al. 1987; Catchpole et al. 1987, 1988, 1989; Whitelock et al. 1988, 1989), 1998A (Pastorello et al. 2005), and 2000cb (Hamuy 2001). The colour curves of the subluminous type IIP SN 2005cs (Tsvetkov et al. 2006; Pastorello et al. 2009) is also shown for comparison.

for classical SNe IIP (about 1 month for SN 2009E, while $\sim 2$ months for type IIP SNe). Between day 30 and day 110, during the recombination phase, the colours remain almost constant. Then, with the post-peak luminosity decline, the SN becomes redder again, reaching $B-V \approx 1.4$ between 110 and 140 days after explosion (this is remarkably similar to the $B-V$ colour observed in SN 1987A). The $V-I$ colour, instead, peaks at about 200 days $(V-I \approx 1.6 \mathrm{mag}$ ), which is a bit earlier than observed for SN $1987 \mathrm{~A}(V-I \approx 1.4$ mag between 230 and 310 days). Subsequently, the colours turn back to the blue again.

The $R$-band absolute light curve of SN 2009E is shown in Fig. 4, together with those of a number of other type II SNe with a broad, delayed light curve peak, similar to that observed for SN 1987A. In analogy with the early discovery of SN 1987A (Arnett et al. 1989), also SN 2009E was likely caught soon after the core-collapse. Marginal evidence of the initial sharp peak due to the shock breakout is possibly visible (Fig. 4, panel C), although the large error bars of the unfiltered amateur images make this finding rather uncertain.

Nevertheless, we can constrain the explosion epoch of SN 2009E with a small uncertainty through a comparison with the early photometric evolution of SN 1987A. Hereafter we will adopt JD $=2454832.5_{-5}^{+2}$ as the best estimate for the corecollapse epoch of SN 2009E. The distances and reddenings used for our sample of 1987A-like events shown in Fig. 4 are listed in Tables 4 and 5. SN 2009E is the faintest SN in this sample at all the epochs. In particular, its peak magnitude $M_{R} \sim-16.2$ is about 0.4 mag fainter than that of SN 1987A and its ${ }^{56} \mathrm{Ni}$ mass, as derived from the luminosity of the radioactive tail relative to that of SN 1987A, is $0.040_{-0.011}^{+0.015} M_{\odot}$, the lowest among the objects in our sample.

Another remarkable property of SN 2009E is that its $R$-band light curve peaks at about $+96 \mathrm{~d}$ after core-collapse, significantly later than SN 1987A ( $\sim 84$ days). This suggests different ejecta parameters for SN 2009E (e.g. smaller initial radius, lower expansion velocity or a more massive envelope, see Sect. 3.2). Interestingly, fainter $\mathrm{SNe}$ in our sample seem to reach peak magnitude later than the luminous objects (see also Appendix B).

\subsection{Spectra}

As mentioned above, the spectroscopic classification of SN 2009E was performed about 80 days after the SN discovery. To our knowledge, no spectra exist to witness the early time evolution of this object. After the SN classification, we started a regular spectroscopic monitoring covering the SN evolution from near maximum light to the nebular phase. Spectra were reduced in the IRAF environment using standard prescriptions. After the optimal extraction, the SN spectra were wavelength calibrated using arc lamp spectra and then flux calibrated using flux standard stars observed in the same nights as the SN. A final check with photometry was performed in order to fine tune the flux calibration of the spectra, with a remaining uncertainty in the flux calibration of less than $10 \%$. Basic information on the spectra collected for SN 2009E is listed in Table 3, while the spectral sequence until August 15, 2009 is shown in Fig. 5.

Since the earliest spectrum was obtained about 83 days after the core-collapse, the continuum is quite red, indicative of relatively low temperatures $\left(T_{\mathrm{bb}} \approx 6000 \mathrm{~K}\right)$. All the photospheric spectra show a clear flux deficit at the blue wavelengths (below 


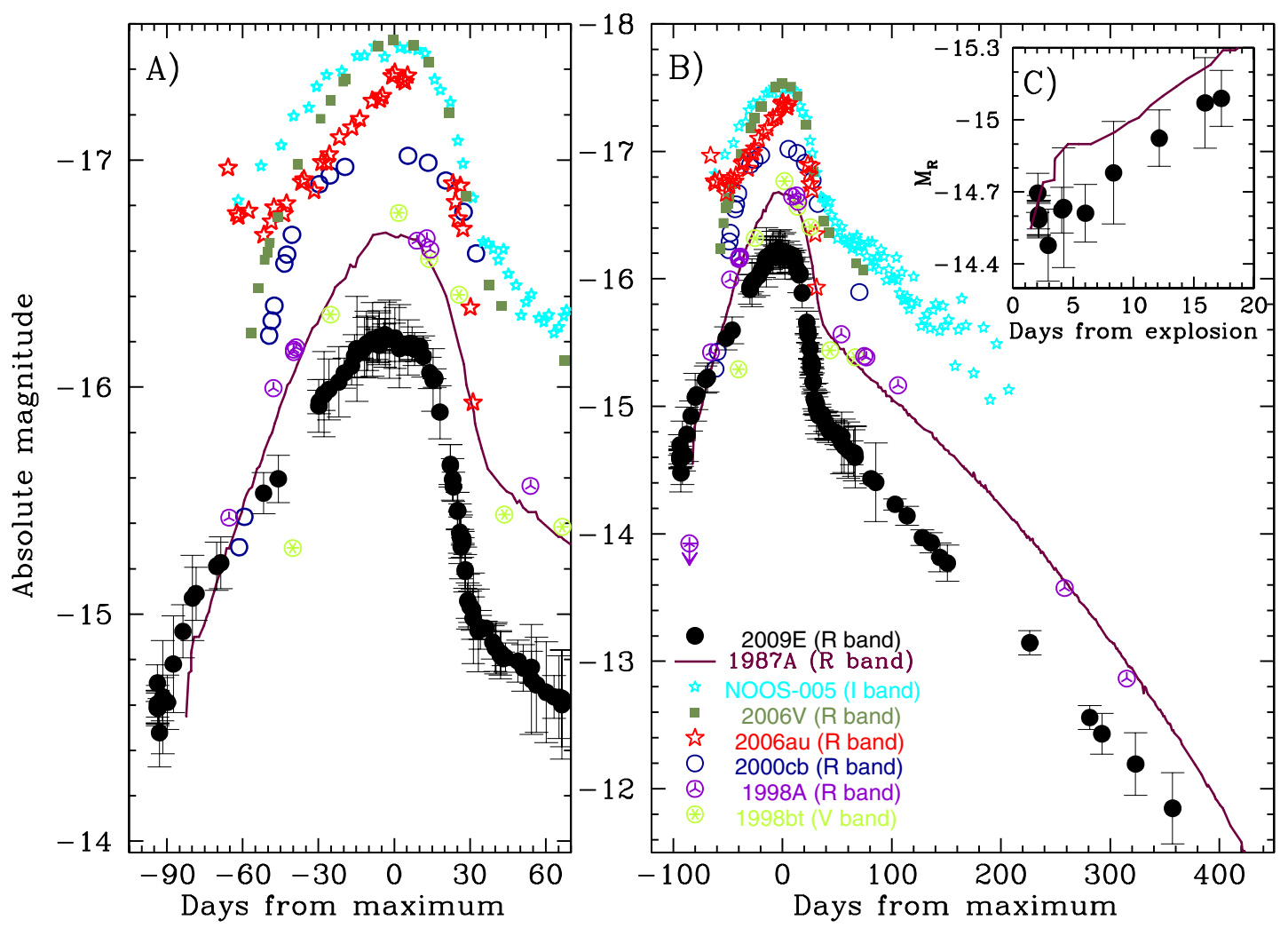

Fig. 4. $R$-band absolute light curve of SN 2009E compared with the absolute light curves of a number of 1987A-like events: NOOS-005 (I band, OGLE collaboration), SNe 2006V, 2006au, 1998bt ( $V$ band, see Germany 1998), 2000cb, 1998A and the prototype 1987A. Panel A): detail on the broad light curve peak. Panel B): the full light curve evolution. Panel C): blow-up of the very early-time light curves of SNe 2009E and 1987A, soon after shock break-out.

Table 4. Parameters of the explosion sites of the 1987A-like SNe.

\begin{tabular}{|c|c|c|c|c|c|c|c|c|}
\hline SN & Host galaxy & Type $^{1}$ & $\overline{\mu \mu^{2}}$ & 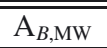 & $\overline{M_{B}}$ & $\overline{\mathrm{d}_{\mathrm{SN}}^{0} / r_{25}}$ & P04 & P04 corr. \\
\hline $1909 \mathrm{~A}$ & NGC 5457 & $\mathrm{SABc}$ & $29.34^{3}$ & 0.037 & -21.01 & $1.11^{9}$ & 8.59 & $8.30^{10}$ \\
\hline $1982 \mathrm{~F}$ & NGC 4490 & SBcd & 29.92 & 0.093 & -20.25 & 0.23 & 8.53 & $8.60^{11}$ \\
\hline 1987A & LMC & $\mathrm{SBm}$ & $18.50^{4}$ & 0.324 & -18.02 & - & 8.31 & - \\
\hline 1998A & IC 2627 & $\mathrm{SABc}$ & 32.19 & 0.518 & -20.03 & 0.66 & 8.51 & 8.41 \\
\hline $1998 \mathrm{bt}$ & A132541-2646 & - & $36.41^{5}$ & 0.244 & -13.25 & - & 7.98 & - \\
\hline $2000 \mathrm{cb}$ & IC 1158 & $\mathrm{SABc}$ & $32.68^{6}$ & 0.491 & -19.34 & 0.93 & 8.46 & 8.24 \\
\hline NOOS-005 & 2MASX J05553978-6855381 & $S$ & 35.46 & $0.656^{8}$ & -20.01 & 1.26 & 8.51 & 8.17 \\
\hline $2004 \mathrm{em}$ & IC 1303 & $\mathrm{Sc}$ & 34.07 & 0.466 & -19.73 & 0.96 & 8.49 & 8.26 \\
\hline 2005ci & NGC 5682 & $\mathrm{Sb}$ & 32.73 & 0.141 & -18.07 & 0.66 & 8.36 & 8.26 \\
\hline $2006 \mathrm{~V}$ & UGC 6510 & $\mathrm{SABc}$ & $34.36^{7}$ & 0.125 & -20.97 & 1.31 & 8.59 & 8.22 \\
\hline $2006 a u$ & UGC 11057 & $\mathrm{Sc}$ & $33.38^{7}$ & 0.742 & -20.64 & 0.90 & 8.56 & 8.36 \\
\hline 2009E & NGC 4141 & $\mathrm{SBc}$ & 32.38 & 0.086 & -17.75 & 0.68 & 8.33 & 8.22 \\
\hline
\end{tabular}

Notes. In Col. 3 we report the morphologic types of the host galaxies, in Cols. 4 and 5 the adopted distance moduli and Galactic extinctions from Schlegel et al. (1998), in Col. 6 the $B$-band absolute magnitudes of the galaxies, in Col. 7 the ratios between the deprojected position of the $\mathrm{SN}$ and $r_{25}$ computed following Hakobyan et al. (2009), in Col. 8 the oxygen abundances from Pilyugin's relation (computed at $0.4 r_{25}$, Pilyugin et al. 2004), in Col. 9 the corrected oxygen abundances from Pilyugin's relation computed at the deprojected SN distance. ${ }^{(1)}$ Morphologic type as quoted by HyperLeda. ${ }^{(2)}$ Unless otherwise specified, distance moduli are computed from HyperLeda's $v_{\mathrm{Vir}}$, adopting $H_{0}=72 \mathrm{~km} \mathrm{~s}^{-1} \mathrm{Mpc}^{-1}$. (3) Distance computed from the tip of the red giant branch (TRGB, Rizzi et al. 2007). Cepheid distances are found to be too sensitive to metallicity. The metallicity correction is even more crucial in the case of NGC 5457 where inner metal-poor and outer metal-rich cepheids give uncorrected distance moduli that may differ by $0.3-0.4$ mag (Saha et al. 2006). ${ }^{(4)}$ For LMC we adopted a cepheid distance, well consistent with that derived from the TRGB (Sakai et al. 2004; Rizzi et al. 2007). ${ }^{(5)}$ SN 1998bt was discovered in the course of the Mount Stromlo Abell cluster supernova search (Germany et al. 2004). Unfortunately, no spectroscopic classification exists for this SN. According to Germany et al. (2004), there is a very faint host galaxy at the position of SN 1998bt, but no spectrum was ever obtained of this galaxy. We assume that the galaxy hosting SN 1998bt belongs to a cluster monitored by the search whose average redshift is $z=0.046 .{ }^{(6)}$ Average EPM distance from Table 4.1 of Hamuy (2001). (7) Distance as in Taddia et al. (2011), but obtained adopting an Hubble Constant $H_{0}=72 \mathrm{~km} \mathrm{~s}^{-1} \mathrm{Mpc}^{-1}$. (8) This galaxy is behind LMC, so the extinction reported here includes both Galaxy and LMC contributions (see text for references). ${ }^{(9)}$ Computed using the position angle provided by Jarrett et al. (2003). ${ }^{(10)}$ Other sources give $12+\log (\mathrm{O} / \mathrm{H}) \approx 7.7-7.8$ at the position of SN 1909A (Kennicutt et al. 2003; Pilyugin et al. 2004; Bresolin 2007). ${ }^{(11)}$ An alternative estimate from Pilyugin \& Thuan $(2007)$ gives $12+\log (\mathrm{O} / \mathrm{H})=8.35$. 


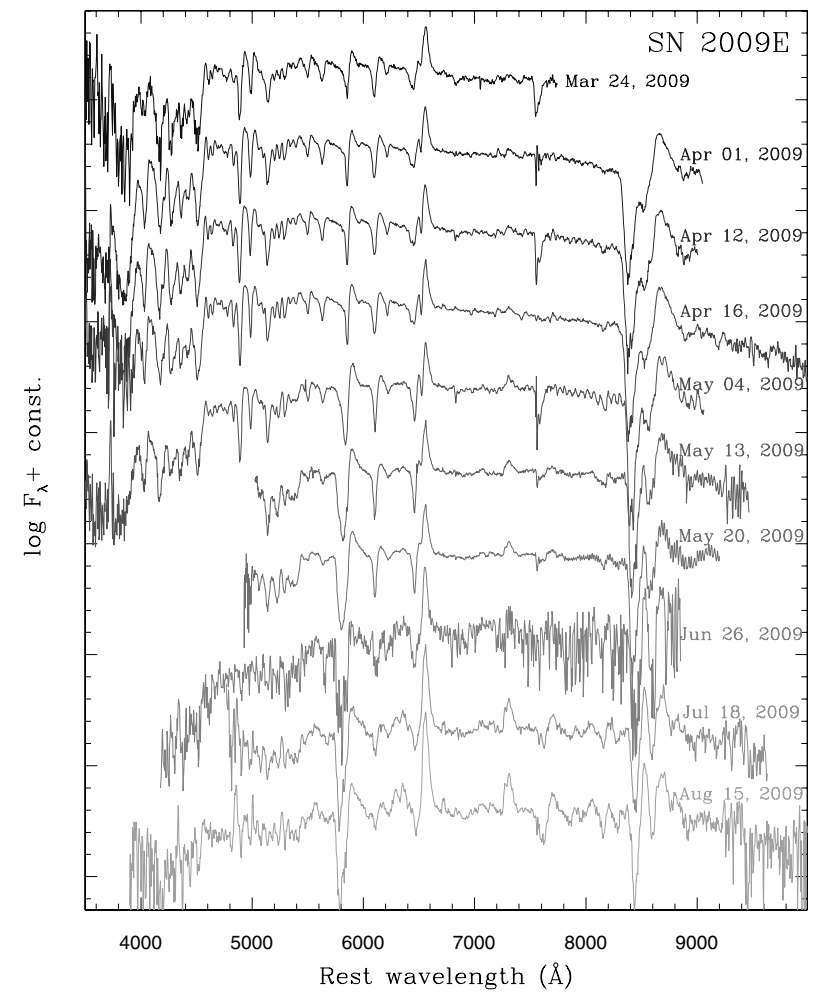

Fig. 5. Spectral sequence for SN 2009E, starting from about 80 days after core-collapse.

$4500 \AA$ ) that is due to line blanketing from increasingly strong metal lines. These spectra are rich in narrow P-Cygni lines indicating remarkably low velocity of the expanding ejecta. From the position of the minima of the Sc II $\lambda 6245$ and $\lambda 5527$ lines, we measured $v_{\mathrm{ph}} \approx 1600 \mathrm{~km} \mathrm{~s}^{-1}$ in our earliest spectrum and about $1000 \mathrm{~km} \mathrm{~s}^{-1}$ at the end of the photospheric phase (Fig. 6). In the same period, the velocity of the unblended Ba II $\lambda 6142$ line declines from $2200 \mathrm{~km} \mathrm{~s}^{-1}$ to $1850 \mathrm{~km} \mathrm{~s}^{-1}$.

In Fig. 7, a late time photospheric spectrum of SN 2009E (phase about 3 months after explosion) is compared with spectra of SN 1987A and the 1987A-like event SN 1998A (Pastorello et al. 2005; see also Fig. 7, top panel), and with spectra of faint SNe IIP (Turatto et al. 1998; Benetti et al. 2001; Pastorello et al. 2004, 2009, see Fig. 7, bottom panel). While the strong Ba II lines are a common feature both for the spectra of SN 1987A and under-luminous, ${ }^{56} \mathrm{Ni}$-poor type IIP SNe, the narrowness of the spectral lines in SN 2009E is more reminiscent of 1997Dlike events (Turatto et al. 1998; Pastorello et al. 2004).

Together with quite a weak $\mathrm{H} \alpha$, we clearly identify prominent lines of Na ID, Ca II, O I, Fe II, Ti II, Sc II and Cr II [see Pastorello et al. (2006) for a detailed line identification for underluminous SNe IIP during the photospheric phase, and Benetti et al. (2001) for the nebular phase]. The Ba II lines (multiplet 2) are among the strongest spectral features. This is a common characteristic of both SN 1987A (Williams 1987; Mazzali et al. 1992; Mazzali \& Chugai 1995) and faint SNe IIP (Pastorello et al. 2004). The presence of prominent Ba II lines in the photospheric spectra of SN 1987A was interpreted by Tsujimoto \& Shigeyama (2001) as a signature of an overabundance of $\mathrm{Ba}$ in the outer layers of the $\mathrm{SN}^{6}$. However, the

\footnotetext{
${ }^{6}$ Note that Utrobin \& Chugai (2005) favoured time-dependent ionization effects to explain the presence of prominent $\mathrm{Ba}$ II lines in photospheric spectra of SN 1987A.
}

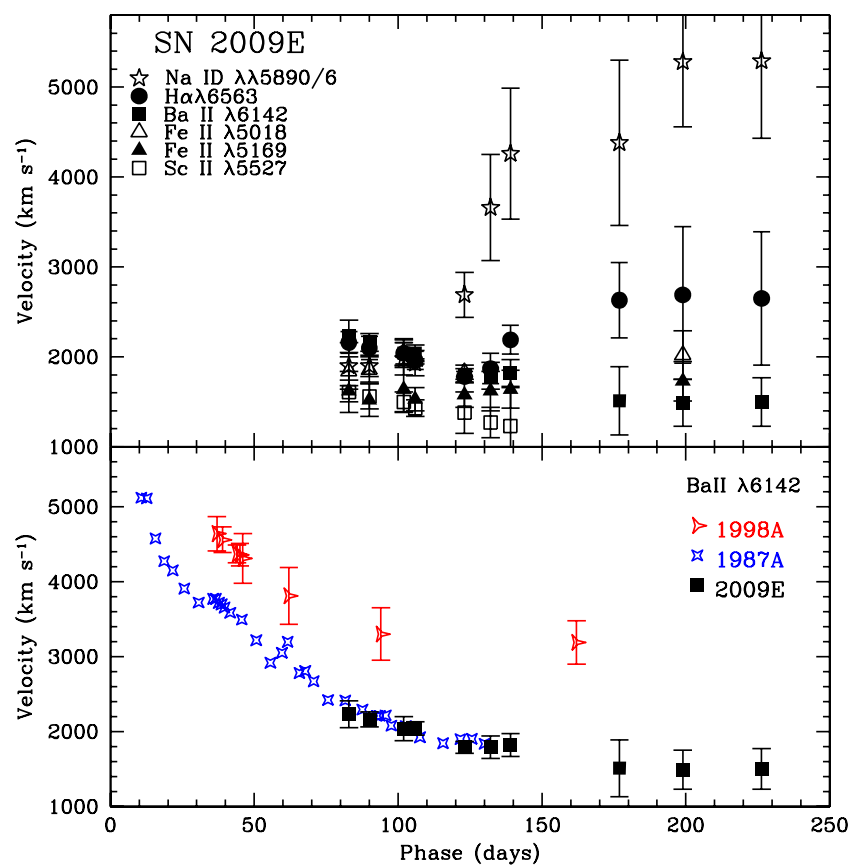

Fig. 6. Top: evolution of the expansion velocities for the principal lines in the spectra of SN 2009E. Bottom: comparison of the velocities derived for the Ba II $\lambda 6142$ line in SNe 2009E, 1987A and 1998A.

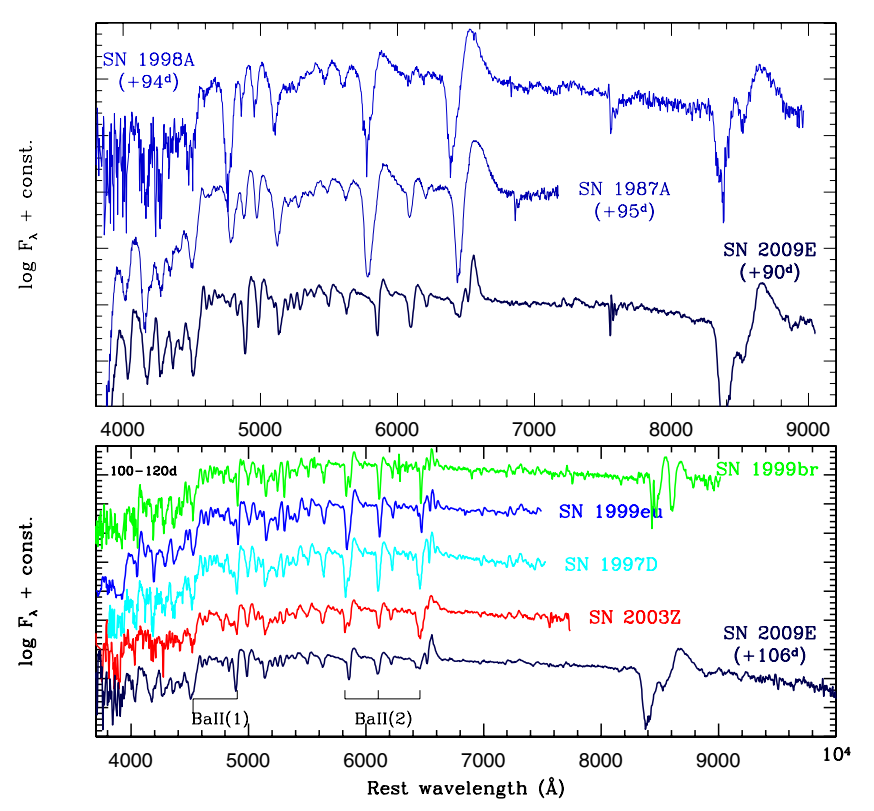

Fig. 7. Top: a spectrum of SN 2009E obtained on Apr. 1st, 2009 is compared with spectra of SNe 1998A (Pastorello et al. 2005) and 1987A (Pun et al. 1995) at a similar phase. Bottom: a post-maximum spectrum of SN 2009E (Apr. 16th, 2009) is compared with spectra of underluminous type IIP SNe obtained around the end of the plateau phase (Turatto et al. 1998; Pastorello et al. 2004).

presence of prominent Ba II features in underluminous SNe IIP can also be explained invoking temperature effects. The evolution of Na ID is puzzling. The absorption component becomes broader with time (see Fig. 5 for the evolution of this spectral feature from 2009 April 16 to May 13). A similar behaviour, although less extreme, is observed also for $\mathrm{H} \alpha$. This effect is probably due to line blending, with the Ba II $\lambda 5854$ and $\lambda 6497$ lines (and possibly of other metal features) having an increasing strength relative to $\mathrm{Na}$ ID and $\mathrm{H} \alpha$ respectively. The evolution 


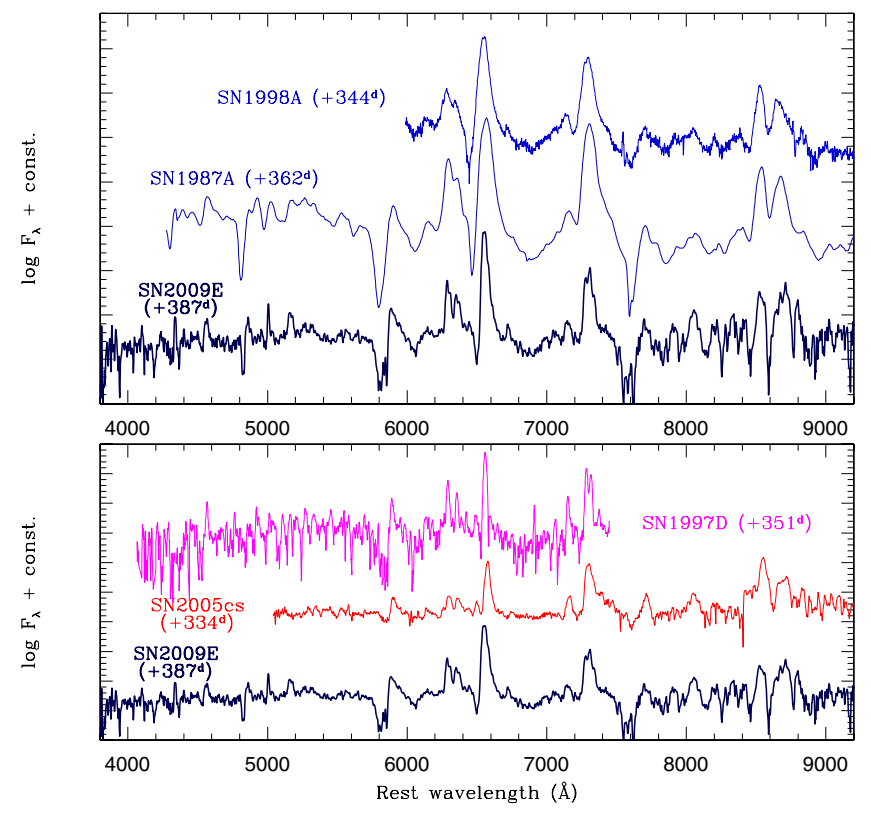

Fig. 8. Top: a nebular spectrum of SN 2009E is compared with spectra of SNe 1998A (Pastorello et al. 2005) and 1987A (Pun et al. 1995) at a similar phase ( $~ 1$ year). The nebular spectrum of SN $2009 \mathrm{E}$ was obtained averaging spectra of 2010 Jan. 22nd and 24th. Bottom: the nebular spectrum of SN 2009E is compared with spectra of underluminous type IIP SNe obtained about 1 year after core-collapse (Benetti et al. 2001; Pastorello et al. 2009).

of the expansion velocities, as deduced from the minima of the P-Cygni of $\mathrm{H} \alpha, \mathrm{Na} \mathrm{I}, \mathrm{Ba}$ II, Fe II and Sc II, is shown in Fig. 6. Metal lines show a monotonic decline and velocities at the end of the plateau phase (1200-1800 $\mathrm{km} \mathrm{s}^{-1}$ ) that are only marginally higher than those observed in sub-luminous type IIP $\mathrm{SNe}\left(800-1200 \mathrm{~km} \mathrm{~s}^{-1}\right.$, see e.g. Turatto et al. 1998; Pastorello et al. 2004, 2009), whilst $\mathrm{H} \alpha$ and $\mathrm{Na}$ ID display an opposite trend. Since May (around 4 months past explosion, Fig. 5), the classical nebular doublets of [Ca II] $\lambda \lambda 7291-7323$ and [O I] $\lambda \lambda$ 6300-6364 become visible, and increase in strength with time.

A late time spectrum of SN 2009E was obtained at the Nordic Optical Telescope (NOT) about 1 year after explosion (see Fig. 8). Although the transition toward a purely nebular appearence is not complete (e.g. broad P-Cygni features of $\mathrm{Na}$ ID, O I $\lambda 7774$ and Ca II NIR are still visible), the spectrum is clearly dominated by prominent $\mathrm{H} \alpha$ in emission, [O I] $\lambda \lambda 6300$, 6364 and [Ca II] $\lambda \lambda 7291,7323$. In addition, a number of [Fe II] features are detected. In this phase the spectrum of SN 2009E more closely resembles those of SN 1987A and SN 1998A (Fig. 8, top), although the spectral lines are significantly narrower than those of other 1987A-like events and are closer to those of sub-luminous, ${ }^{56} \mathrm{Ni}$-poor $\mathrm{SNe}$ (Fig. 8, bottom).

\section{Constraining the progenitor of SN 2009E}

\subsection{The ejected oxygen mass}

The strength of the $[\mathrm{O}$ I] $\lambda \lambda 6300,6364$ lines in the nebular spectra of core-collapse $\mathrm{SNe}$ can be used to roughly estimate the $\mathrm{O}$ mass ejected in the SN explosion (Uomoto 1986; Li \& McCray 1992; Chugai 1994). This method has been applied for determining the $\mathrm{O}$ masses for a few type IIP SNe (see Maguire et al. 2010 , and references therein). We first make an attempt to estimate the minimum $\mathrm{O}$ mass needed to produce the $[\mathrm{O} \mathrm{I}] \lambda \lambda$ 6300-6364 feature with the relation between the $\mathrm{O}$ mass and the total flux of the doublet presented by Uomoto (1986). Using the nebular spectrum of SN 2009E, we measure an observed flux $F_{[\mathrm{OI}]} \approx 3.35 \times 10^{-15} \mathrm{erg} \mathrm{s}^{-1} \mathrm{~cm}^{-2}$. Accounting for the reddening and distance estimates as mentioned in Sect. 2.1, we obtain for SN 2009E a minimum O mass in the range between $0.05 M_{\odot}$ and $0.22 M_{\odot}$ (assuming the extreme values of $3500 \mathrm{~K}$ and $4500 \mathrm{~K}$, respectively as the temperature of the O-rich material, like in Maguire et al. 2010). As a comparison, Maguire et al. (2010) derived a minimum $\mathrm{O}$ mass range for SN 1987A of 0.3-1.1 $M_{\odot}$. The only other 1987A-like object with nebular spectra available is SN 1998A (Pastorello et al. 2005). Using the spectrum at phase $\sim 344$ days (see Fig. 8, top), we obtain an O mass in the range $0.24-1.01 M_{\odot}$.

In order to improve the $\mathrm{O}$ mass estimate, we adopt the method already used in Elmhamdi et al. (2003), which is based on the fact that the luminosity of the [O I] doublet in the nebular phase is known to be powered by the $\gamma$-rays produced in the radioactive decays of ${ }^{56} \mathrm{Co}$ to ${ }^{56} \mathrm{Fe}$, and deposited in the O-rich ejecta. Since the mass of O in SN 1987A is reasonably wellknown (1.2-1.5 $M_{\odot}$, Li \& McCray 1992; Chugai 1994; Kozma \& Fransson 1998), we can derive the O masses of SNe 2009E and 1998A via comparison with the O mass of SN 1987A. We use the relation linking the $\mathrm{O}$ mass with the luminosities of the $[\mathrm{O} \mathrm{I}]$ doublet $^{7}$ and of the radioactively powered light curve tail, computed at similar phases (Elmhamdi et al. 2003):

$L_{[\mathrm{OI}]}=\eta_{\mathrm{O}} \frac{M_{\mathrm{O}}}{M_{\mathrm{exc}}} L_{56} \mathrm{Co}$,

where $L_{[\mathrm{OI}]}$ and $L_{56} \mathrm{Co}$ are the luminosities of the [O I] doublet and of the radioactive tail (respectively), $M_{\mathrm{O}}$ is the mass of $\mathrm{O}$, $M_{\text {exc }}$ represents the whole excited mass in which the radioactive energy is deposited, and $\eta_{\mathrm{O}}$ is the efficiency of the transformation of the deposited energy into [O I] line radiation. Assuming that $M_{\text {exc }}$ and $\eta_{0}$ are similar in all 1987A-like events and noting that (since the late light curve of SN 2009E follows the ${ }^{56}$ Co decay rate)

$\frac{L_{56} \mathrm{Co}_{0}(1987 A)}{L_{56} \mathrm{Co}(S N)} \propto \frac{M_{56} \mathrm{Ni}(1987 A)}{M_{56 \mathrm{Ni}}(S N)}$,

from Eq. (1), we obtain:

$M_{\mathrm{O}}(S N)=M_{\mathrm{O}}(1987 A) \times \frac{L_{[\mathrm{OI}]}(S N) \times M_{56 \mathrm{Ni}}(1987 A)}{L_{[\mathrm{OI}]}(1987 A) \times M_{56} \mathrm{Ni}(S N)}$.

Now we have all the ingredients needed to estimate the $\mathrm{O}$ mass in SNe 2009E and 1998A from Eq. (3) using the information available for SN 1987A (Li \& McCray 1992; Chugai 1994). For SN 2009E we obtain $M_{\mathrm{O}} \approx 0.60-0.75 M_{\odot}$ (using as $\mathrm{O}$ mass for SN 1987A the extreme values of $M_{\mathrm{O} \text {,min }}=1.2 M_{\odot}$ and $M_{\mathrm{O}, \max }=1.5 M_{\odot}$, respectively), while for SN $1998 \mathrm{~A}$ we obtain $M_{\mathrm{O}} \approx 1.18-1.48 M_{\odot}$. Therefore, with this method, we determine for the under-luminous SN 2009E an ejected mass of O that is a factor of 2 smaller than that of SN 1987A (note that for SN 2009E also the ${ }^{56} \mathrm{Ni}$ mass is a factor of $\sim 2$ smaller). In the case of SN 1998A we obtain an O mass that matches the values of SN 1987A, despite the former object ejected more ${ }^{56} \mathrm{Ni}$.

According to the models presented by Woosley \& Weaver (1995) and Thielemann et al. (1996), a star with main sequence mass of about $18-20 M_{\odot}$ produces $1.2-1.5 M_{\odot}$ of oxygen and a

\footnotetext{
7 We note that the luminosity of the [O I] doublet in SN 1987A and type IIP SNe remains almost constant over a long period during the nebular phase (between $\sim 200-400$ days, see e.g. Fig. 8 in Elmhamdi et al. 2003).
} 

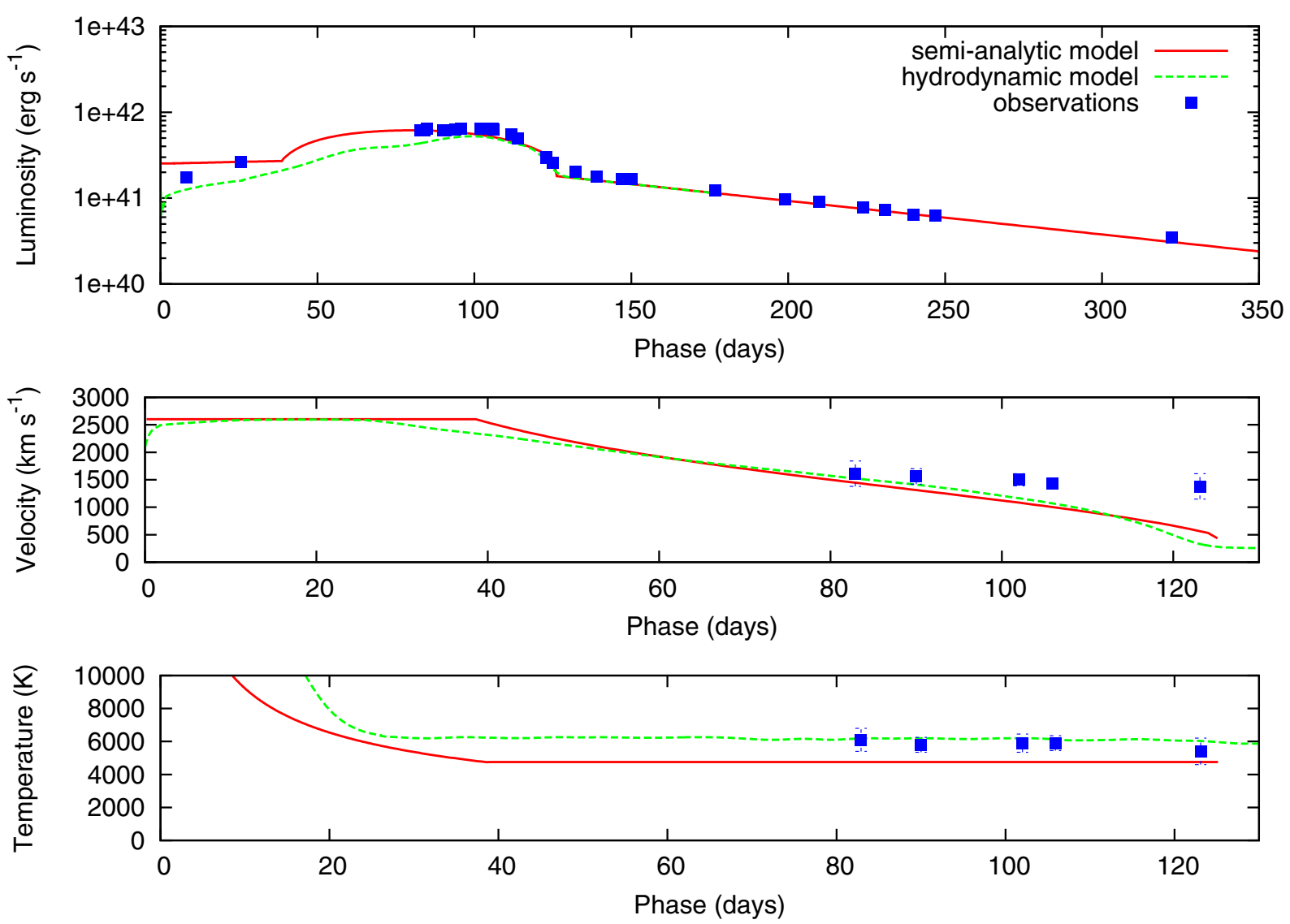

Fig. 9. Comparison of the evolution of the main observables of SN 2009E with the best-fit model computed with the relativistic radiationhydrodynamics code (total energy 0.6 foe, initial radius $7 \times 10^{12} \mathrm{~cm}$, envelope mass $19 M_{\odot}$ ). The best-fit model computed with the semi-analytic code (total energy 1.3 foe, initial radius $6 \times 10^{12} \mathrm{~cm}$, envelope mass $26 M_{\odot}$ ) is also shown. Top, middle, and bottom panels show respectively the bolometric light curve, the photospheric velocity, and the photospheric temperature as a function of time. As a tracer for the photospheric velocity we measured the positions of the minimum of the P-Cygni profiles of the Sc II lines.

${ }^{56} \mathrm{Ni}$ mass of $0.07-0.1 M_{\odot}$. This is in excellent agreement with the values observed in SNe 1987A and 1998A. It is more difficult to provide an interpretation for the abundances displayed by SN 2009E (both $M_{\mathrm{O}}$ and $M_{56} \mathrm{Ni}$ are smaller by a factor 2 than those of SN 1987A), although we may tentatively guess that the lower $\mathrm{O}$ and ${ }^{56} \mathrm{Ni}$ masses would point toward a lower-mass main sequence star. We remark that the above estimates have been obtained under the simplifying assumption that $M_{\mathrm{exc}}$ and $\eta_{\mathrm{O}}$ were the same for SNe 1987A, 1998A and 2009E. This is not necessarily true, since we can reasonably expect a range of values for these parameters in 1987A-like events, and this would significantly affect our $\mathrm{O}$ mass estimates.

\subsection{Modelling the data of SN $2009 E$}

Other physical properties of SN 2009E (namely, the ejected mass, the progenitor initial radius and the explosion energy) have been estimated by performing a model vs. data comparison using two codes that compute the bolometric light curve, the evolution of the ejecta velocity and the continuum temperature at the photosphere of a SN. The first code, based on a simplified semianalytic treatment of the ejecta evolution (Zampieri et al. 2003), is used to perform a preparatory study in order to constrain the parameter space. The second code, that includes a more accurate treatment of radiative transfer and radiation hydrodynamics (Pumo et al. 2010; Pumo \& Zampieri 2011), computes the tighter grid of models needed for the final comparison. This is similar to the procedure adopted for SN 2007od in Inserra et al. (2011).

The bolometric light curve was computed from the observed multicolor light curves using the assumptions on extinction and distance modulus reported in Sect. 2.1. We have adopted the explosion epoch estimated in Sect. $2.3\left(\mathrm{JD}=2454832.5_{-5}^{+2}\right)$. Finally, we have assumed that SN 2009E had the same color evolution as SN 1987A (this is a reasonable assumption, see e.g. Fig. 3), and hence

$L(2009 E)=L(1987 A) \times \frac{L_{B V R I}(2009 E)}{L_{B V R I}(1987 A)}$,

where $L$ and $L_{B V R I}$ are the bolometric and quasi-bolometric (BVRI) luminosities of the two $\mathrm{SNe}$ (bolometric data for $\mathrm{SN}$ 1987A were taken from Catchpole et al. 1987, 1988).

The best $\chi^{2}$ fit to the bolometric light curve of SN 2009E obtained with the full radiation-hydrodynamics code returned values of a total (kinetic plus thermal) energy $(E)$ of 0.6 foe, an initial radius $\left(R_{0}\right)$ of $7 \times 10^{12} \mathrm{~cm}$, and an envelope mass $\left(M_{\mathrm{ej}}\right)$ of $19 M_{\odot}$ (see Fig. 9). As a comparison, the fit with the semi-analytic code gave $E=1.3$ foe, $R_{0}=6 \times 10^{12} \mathrm{~cm}$, and $M_{\mathrm{ej}}=26 M_{\odot}$ (see again Fig. 9 ). The parameters inferred from the two best-fits are in reasonable agreement. The envelope mass (and consequently the total energy) are slightly overestimated by the semi-analytic code as a consequence of the different mass distribution. Indeed, given the assumption of uniform density throughout the ejecta, the semi-analytic code tends 
Table 5. Adopted parameters for 1987A-like SNe.

\begin{tabular}{|c|c|c|c|c|c|c|c|c|c|}
\hline SN & $E_{\mathrm{tot}}(\mathrm{B}-\mathrm{V})^{a}$ & $\mathrm{JD}_{\text {expl. }}$ & Band & $\mathrm{JD}_{\text {peak }}$ & $t-t_{0}$ (days) & $m_{\text {peak }}($ filter $)$ & $M_{\text {peak }}$ & $M\left({ }^{56} \mathrm{Ni}\right)$ & Sources \\
\hline 1909A & 0.009 & $2418310 \pm 30$ & $B$ & $2418383 \pm 7$ & $73 \pm 31$ & $14.47 \pm 0.30$ & -15.91 & $0.13 M_{\odot}^{b}$ & 1 \\
\hline \multirow[t]{2}{*}{$1982 \mathrm{~F}$} & 0.022 & $2445014 \pm 15$ & $B$ & $2445087 \pm 4$ & $73 \pm 16$ & $16.40 \pm 0.02$ & -13.61 & - & $2,3,4$ \\
\hline & & & V & $2445097 \pm 3$ & $83 \pm 15$ & $14.95 \pm 0.10$ & -15.04 & & \\
\hline \multirow[t]{4}{*}{ 1987A } & 0.19 & $2446849.816^{c}$ & $B$ & $2446932.0 \pm 1.1$ & $82.2 \pm 1.1$ & $4.52 \pm 0.01$ & -14.77 & $0.075 M_{\odot}$ & 5 \\
\hline & & & V & $2446932.2 \pm 1.0$ & $82.4 \pm 1.0$ & $2.96 \pm 0.01$ & -16.13 & & \\
\hline & & & $R$ & $2446933.6 \pm 1.0$ & $83.8 \pm 1.0$ & $2.27 \pm 0.01$ & -16.67 & & \\
\hline & & & $I$ & $2446935.2 \pm 0.9$ & $85.4 \pm 0.9$ & $1.90 \pm 0.01$ & -16.88 & & \\
\hline \multirow[t]{4}{*}{ 1998A } & 0.120 & $2450801 \pm 4$ & $B$ & $2450876.3 \pm 7.4$ & $75.3 \pm 8.4$ & $17.59 \pm 0.13$ & -15.12 & $0.09 M_{\odot}$ & 6 \\
\hline & & & $V$ & $2450885.4 \pm 8.3$ & $84.4 \pm 9.2$ & $16.31 \pm 0.09$ & -16.28 & & \\
\hline & & & $R$ & $2450885.6 \pm 3.9$ & $84.6 \pm 5.6$ & $15.73 \pm 0.06$ & -16.78 & & \\
\hline & & & $I$ & $2450889.2 \pm 5.5$ & $88.2 \pm 6.8$ & $15.37 \pm 0.07$ & -17.05 & & \\
\hline \multirow{2}{*}{$1998 \mathrm{bt}$} & 0.057 & $2450831 \pm 30$ & V & $2450905 \pm 6$ & $74 \pm 31$ & $19.81 \pm 0.05$ & -16.79 & & 7,0 \\
\hline & & & $R$ & $2450908 \pm 8$ & $77 \pm 31$ & $19.38 \pm 0.08$ & -17.18 & & 8,0 \\
\hline \multirow[t]{4}{*}{$2000 \mathrm{cb}$} & 0.112 & $2451653.8 \pm 1.4$ & $B^{d}$ & & & $18.23 \pm 0.02$ & -14.94 & $0.11 M_{\odot}$ & $8,9,10$ \\
\hline & & & $V$ & $2451714.6 \pm 4.7$ & $60.8 \pm 4.9$ & $16.58 \pm 0.02$ & -16.47 & & \\
\hline & & & $R$ & $2451725.2 \pm 2.1$ & $71.4 \pm 2.5$ & $15.95 \pm 0.02$ & -17.03 & & \\
\hline & & & $I$ & $2451729.0 \pm 1.4$ & $75.2 \pm 2.0$ & $15.56 \pm 0.02$ & -17.34 & & \\
\hline NOOS-005 & 0.160 & $2452855 \pm 15$ & $I$ & $2452939.5 \pm 2.7$ & $84.5 \pm 15.2$ & $18.22 \pm 0.02$ & -17.51 & $0.13 M_{\odot}$ & 0 \\
\hline $2005 \mathrm{ci}$ & $0.033 ?$ & $2453521 \pm 3$ & $u n f$. & & & $<17.5$ & $<-15.3$ & & 10 \\
\hline $2006 \mathrm{~V}^{e}$ & 0.029 & $2453748 \pm 4$ & $B$ & 2453823.7 & 75.7 & $18.29 \pm 0.01$ & -16.20 & $0.13 M_{\odot}$ & 11 \\
\hline $2006 a u^{e}$ & 0.312 & $2453794 \pm 9$ & $B$ & 2453865.5 & 71.5 & $18.63 \pm 0.02$ & -16.06 & $<0.07 M_{\odot}$ & 11 \\
\hline \multirow[t]{4}{*}{$2009 \mathrm{E}$} & 0.04 & $2454832.5_{-5}^{+2}$ & $B$ & $2454919.0 \pm 6.5$ & $86.5 \pm 6.8$ & $18.01 \pm 0.03$ & -14.54 & $0.04 M_{\odot}$ & 0 \\
\hline & & & V & $2454924.9 \pm 5.9$ & $92.4 \pm 6.2$ & $16.76 \pm 0.04$ & -15.74 & & \\
\hline & & & $R$ & $2454928.3 \pm 2.8$ & $95.8 \pm 3.4$ & $16.25 \pm 0.02$ & -16.21 & & \\
\hline & & & $I$ & $2454928.3 \pm 6.0$ & $95.8 \pm 6.3$ & $15.98 \pm 0.03$ & -16.46 & & \\
\hline
\end{tabular}

Notes. The total extinction is reported in Col. 2, the JD of explosion in Col. 3, the bands in Col. 4, the JD of the maximum light in Col. 5, the phase of the broad maximum (in days past explosion) in Col. 6, the apparent and absolute peak magnitudes in Cols. 7 and 8 (respectively) and the ${ }^{56} \mathrm{Ni}$ mass in Col. 9. The extinction law of Cardelli et al. (1989) was used in these estimates. The explosion epochs have been determined through a comparison with the light curves of SN 1987A. ${ }^{(a)}$ The SN extinction is computed accounting for both Milky Way (Schlegel et al. 1998, see also Column 3 of Table 4) and host galaxy reddening components; in the case of NOOS-005, since the host galaxy is projected behind LMC, we also accounted for the contribution of LMC. ${ }^{(b)}$ Computed using the $B$-band detections at days +429 and +432 , and assuming the same colour evolution as SN 1987A. Using the detection at day +216 , we would obtain $M\left({ }^{56} \mathrm{Ni}\right) \approx 0.35 M_{\odot} .{ }^{(c)}$ Alexeyev \& Alexeyeva $(2008)$ and references therein. (d) The broad delayed maximum typical of 1987A-like SNe is not visible in the $B$ band; here we report the magnitude of the pseudo-plateau visible after the early time $B$-band maximum. ${ }^{(e)}$ For a comprehensive information on the multi-band light curve parameters, see Table 7 of Taddia et al. (2011).

References. 0 = This paper; $1=$ Sandage $\&$ Tammann (1974); $2=$ Yamagata $\&$ Iye (1982); $3=$ Tsvetkov (1984); $4=$ Tsvetkov (1988); $5=$ Whitelock et al. (1989) (and references therein); 6 = Pastorello et al. (2005); 7 = Germany et al. (2004); 8 = Hamuy (2001); $9=$ Hamuy \& Pinto (2002); 10 = Kleiser et al. (2011); 11 = Taddia et al. (2011).

to gather more mass (and hence more energy) in the external, faster-moving layers. The ${ }^{56} \mathrm{Ni}$ distribution also affects the mass estimate because, as a consequence of the simplified uniform distribution adopted in the semi-analytic code, less energy is released at the end of the photospheric phase and, hence, a more massive envelope is needed to sustain the recombination phase.

The ejected ${ }^{56} \mathrm{Ni}$ mass of $0.039 M_{\odot}$ that is adopted in the semi-analytic code is in an excellent agreement with that estimated in Sect. 2.3, whilst the one inferred from the radiation-hydrodynamic code is slightly higher $\left(0.043 M_{\odot}\right)$. Since the latter code accounts also for the amount of material which (eventually) falls back onto the central remnant during the post-explosive evolution, the numerical simulations need a larger initial ${ }^{56} \mathrm{Ni}$ mass to reproduce the observed late-time light curve of SN 2009E.

In Fig. 9 the evolutions of the photospheric velocity and temperature are also shown. The agreement between models and observations in the late photospheric phase is good, with possibly some discrepancy in matching the photospheric velocities at later times ( $\gtrsim 4$ months). The decline of the model velocity profile is faster than that inferred from the observations, probably because some extra radioactive decay heating occurs in the inner part of the ejecta of SN 2009E as a consequence of a different (i.e. more centrally condensed) distribution of ${ }^{56} \mathrm{Ni}$.
Finally, adopting a mass of the compact remnant of about $2 M_{\odot}$ (and assuming a negligible pre-SN mass loss), we derive a final mass of $21 M_{\odot}$ for the progenitor of SN 2009E, which is slightly higher than our rough estimate obtained throught the O mass estimate (Sect. 3.1).

\section{SN 2009E and other 1987A-like events}

Although 1987A-likeness was claimed for a number of objects in the past (e.g. SNe 1923A, 1948B and 1965L, Schmitz \& Gaskell 1988; Young \& Branch 1988; van den Bergh \& McClure 1989), reasonable observational evidence exists for only a small number of events ${ }^{8}$. Additionally, for a few of them [SN 2004em, Gal Yam et al. (2007); SN 2005ci, Arcavi et al. (2009)] data

\footnotetext{
8 Another type II SN, 2004ek, may show some similarity with this SN sub-group. Its light curve, published by Tsvetkov (2008), is rather peculiar, showing a sharp early-time peak, mostly visible in the bluer bands, followed by a pseudo-plateau lasting a couple of months. However, especially in the redder bands, a sort of delayed broad peak is visible, analogous to the one characterizing SNe $1982 \mathrm{~F}$ and $2000 \mathrm{cb}$. As major differences, SN 2004ek is significantly brighter $\left(M_{V} \approx-18\right)$ and its spectrum, showing an $\mathrm{H} \alpha$ mostly in emission (Filippenko et al. 2004) is rather peculiar. For this reason SN 2004ek has been rejected from our sample.
} 
are not published yet or are incomplete. Our limited 1987Alike sample includes the objects that are briefly described in Appendix A (and whose light curves are shown in Appendix B). The sample has been selected among objects that are spectroscopically classified as type II (without showing type IIn-like spectral features), and with light curves showing a slow rise to maximum.

Main parameters of the host galaxies of our sample of 1987A-like events are listed in Table 4. Remarkably, most SNe in our sample occured in late-type galaxies (Scd or irregular). In addition, some of them were hosted by intrinsically faint (dwarf) galaxies, which are thought to have low-metallicities (see e.g. Young et al. 2010). However, at least half of the objects of our sample occurred in luminous spirals. Although luminous spirals are believed to be rather metal-rich, the 1987A-like events hosted in such galaxies occurred in peripheral -and hence more metal deficient- regions of the galaxies. Environments with lowto-moderate metallicity (slightly sub-solar) appear to be a fairly common characteristic for 1987A-like objects.

In Table 5 we report the values of some observational parameters for our $\mathrm{SN}$ sample. The magnitudes of the broad peaks seem to be correlated with the ejected ${ }^{56} \mathrm{Ni}$ masses (i.e. the objects with the brightest peak magnitudes are more ${ }^{56} \mathrm{Ni}-$ rich). The ${ }^{56} \mathrm{Ni}$ masses reported in Table 5 have been computed through a comparison between the late-time uvoir light curves of the different SNe with that of SN 1987A. The bolometric corrections were computed with reference to SN 1987A. The derived ${ }^{56} \mathrm{Ni}$ masses span a range between about $0.04 M_{\odot}$ and $0.13 M_{\odot}{ }^{9}$, similar to that observed in normal type IIP SNe, although cases of extremely ${ }^{56} \mathrm{Ni}$-poor $1987 \mathrm{~A}$-like events (with ${ }^{56} \mathrm{Ni}$ masses of the order of $10^{-3} M_{\odot}$, Pastorello et al. 2004) have not been found so far.

Unfortunately, the sub-sample of 1987A-like SNe with an extensive data sets necessary for modelling is very small. This is necessary to constrain the properties of the progenitor stars. The rather large ejected mass, the moderate amount of synthesized ${ }^{56} \mathrm{Ni}$, and the small initial radius found for SN 2009E (Sect. 3.2) fit reasonably well within the framework of a relatively compact and massive progenitor, and are in a good agreement with the results found in previous works on 1987A-like events (see Table 8 in Taddia et al. 2011). Using an older version of the semi-analytic modelling code than the one adopted here, Pastorello et al. (2005) obtained the following estimates for $\mathrm{SNe}$ 1987A and 1998A: $E(1987 \mathrm{~A})=1.6$ foe, $E(1998 \mathrm{~A})=5.6$ foe; $M_{\mathrm{ej}}(1987 \mathrm{~A})=18 M_{\odot}, M_{\mathrm{ej}}(1998 \mathrm{~A})=22 M_{\odot} ; M_{56 \mathrm{Ni}}(1987 \mathrm{~A})$ $=0.075 M_{\odot}, M_{56 \mathrm{Ni}}=0.11 M_{\odot} ; R_{0}(1987 \mathrm{~A}) \gtrsim 5 \times 10^{12} \mathrm{~cm}$, $R_{0}(1998 \mathrm{~A}) \gtrsim 6 \times 10^{12} \mathrm{~cm}$. The major difference lies in the ejected ${ }^{56} \mathrm{Ni}$ masses of SNe 1987A and 1998A, which are $\times 2$ and $\times 3$ larger (respectively) than the one we have derived for SN 2009E (Sect. 3.2). Explosion parameters for a few additional 1987A-like $\mathrm{SNe}$ are also available in the literature (see also Taddia et al. 2011). Using a one-dimensional Lagrangian radiation-hydrodynamics code, Kleiser et al. (2011) derived the following parameters for the luminous 1987A-like SN 2000cb: $E(2000 \mathrm{cb})=2$ foe, $M_{\mathrm{ej}}(2000 \mathrm{cb})=17.5 M_{\odot}, M_{56 \mathrm{Ni}}(2000 \mathrm{cb})=$ $0.10 M_{\odot}$ and $R_{0}(2000 \mathrm{cb})=3 \times 10^{12} \mathrm{~cm}$. These values are slightly different from those derived by Utrobin \& Chugai (2011) by modelling the spectroscopic and photometric data of SN 2000cb with a different hydrodynamic code. They inferred a presupernova radius of $2.4 \times 10^{12} \mathrm{~cm}$, an ejected mass of $22.3 M_{\odot}$, an

\footnotetext{
9 The lack of photometric points during the nebular phase did not allow us to obtain reliable estimates for the ${ }^{56} \mathrm{Ni}$ masses of SN 1982F.
}

energy of 4.4 foe, and a mass of radioactive ${ }^{56} \mathrm{Ni}$ of $0.083 M_{\odot}{ }^{10}$. With a semi-analytic approach, Taddia et al. (2011) estimated relevant physical parameters for two 1987A-like events, $\mathrm{SNe}$ $2006 \mathrm{~V}$ and $2006 \mathrm{au}: E(2006 \mathrm{~V})=2.4$ foe, $E(2006 \mathrm{au})=3.2$ foe; $M_{\mathrm{ej}}(2006 \mathrm{~V})=17.0 M_{\odot}, M_{\mathrm{ej}}(2006 \mathrm{au})=19.3 M_{\odot} ; M_{56 N i}(2006 \mathrm{~V})$ $=0.127 M_{\odot}, M_{56 \mathrm{Ni}}(2006 \mathrm{au}) \leq 0.073 M_{\odot} ; R_{0}(2006 \mathrm{~V})=5.2 \times$ $10^{12} \mathrm{~cm}, R_{0}(2006 \mathrm{au})=6.3 \times 10^{12} \mathrm{~cm}$.

It is evident that 1987A-like SN explosions have similar kinetic energies (a few $\times 10^{51} \mathrm{erg}$ ) and ejected masses (17 to $\left.22 M_{\odot}\right)$, whilst they appear to span a factor of 3 in ${ }^{56} \mathrm{Ni}$ masses (see also Table 8 in Taddia et al. 2011, for a summary of the parameters of the best-studied 1987A-like events). It is intriguing to note that the modelling reported above suggest that all the 1987A-like SNe would be produced by the explosions of BSG progenitors that have initial radii ranging between 35 and $90 R_{\odot}$, and with final masses around $20 M_{\odot}$. These progenitor masses are systematically higher than those estimated for the RSG progenitors of classical SNe IIP (e.g. Smartt et al. 2009).

\section{Rate of 1987A-like events}

While it would be of interest to know the relative rate of 1987Alike events vs. normal type II SNe, the heterogeneous properties of this class and the uncertainties in the sample population from which the events reported in Table 4 are extracted prevent us from providing any accurate estimates. With the aim to derive an educated guess, the completeness of the SN searches with respect to the 1987A-like objects list can be assessed by comparing the distance modulus distribution of 1987A-like events with that of a suitable reference sample. For the latter, we extracted from the Asiago Supernova Catalogue the list of all type II SNe. If we limit the distance modulus to about $35 \mathrm{mag}$ (at higher redshift incompleteness appears to be significant) it seems that 1987Alike events would account for less than $1.5 \%$ of all type II SNe. This value, that can be considered as a lower limit for the relative rate of 1987A-like events, is very similar to that derived by Kleiser et al. (2011) based on the two 1987A-like SNe discovered by the LOSS.

However, the identification of 1987A-like events demands a fairly good light curve coverage that is not available for many of the type II SNe listed in the catalogue. Actually, reasonably good light curves of type II (IIP, IIL, IIb, IIn) SNe exist for about 300 objects. This approximate estimate was obtained including both SNe whose data have been published in literature, and SNe spectroscopically classified whose photometric follow-up information is available in public pages of current SN monitoring programs ${ }^{11}$. From this estimate, we infer that 1987A-like events comprise about $4 \%$ of all type II SNe. This has to be considered as a sort of upper limit, since we expect that the astronomical community has been investing more efforts to follow peculiar 1987A-like SNe rather than other type II SN sub-types because of their intrinsical rarity. Therefore, we estimate that SNe photometrically similar to SN 1987A comprise between $\sim 1.4$ and $4 \%$ of all type II SNe, corresponding to about $1-3 \%$ of all core-collapse $\mathrm{SNe}$ in a volume-limited sample (e.g. Smartt et al. 2009; Li et al. 2011).

${ }_{10}$ Note, however, that the distance moduli adopted by Pastorello et al. (2005) for the host of SN 2009E ( $\mu=32.41)$ and by Kleiser et al. (2011) and Utrobin \& Chugai (2011) for the host of SN 2000cb $(\mu=32.39)$ are different from those adopted here and reported in Table 4.

11 For example, the ESO/TNG large program on Supernova Variety and Nucleosynthesis Yields (http://graspa.oapd.inaf.it/), the Caltech Core-Collapse Program (http://www . astro. caltech.edu/ avishay/cccp.html) or the Carnegie Supernova Project (http:// csp1.lco.cl/ cspuser1/PUB/CSP.html) 


\section{Summary}

New data for the peculiar type II SN 2009E have been presented. Its light curve is very similar to that of SN 1987A, with a few main differences: the light curve peak is shifted in phase by about +10 days with respect to that of SN 1987A, its luminosity is fainter than that of SN 1987A and the amount of ${ }^{56} \mathrm{Ni}$ deduced from the luminosity of the radioactive tail is about $0.04 M_{\odot}$. This is the lowest ejected ${ }^{56} \mathrm{Ni}$ mass estimated for any $1987 \mathrm{~A}-$ like event studied so far. The spectra of SN 2009E appear to be different from those of SN 1987A (and similar events), while they share major similarities with those of sub-luminous, ${ }^{56} \mathrm{Ni}$-poor SNe IIP (e.g. SN 2005cs) in terms of line velocities and relative line strengths.

In analogy with what has been deduced for SN 1987A, also the observed properties of SN 2009E are consistent with those expected from the explosion of a BSG star. Modelling the data of SN 2009E, we obtained a relatively under-energetic explosion ( $E=0.6$ foe $)$, an initial radius of of $7 \times 10^{12} \mathrm{~cm}$ and an ejected mass of $19 M_{\odot}$, pointing toward a relatively high-mass precursor star $\left(21 M_{\odot}\right)$, very similar to the BSG progenitor inferred for SN 1987A.

Finally, we compared the observed properties of a dozen of 1987A-like SNe available in the literature, and found that this subgroup of type II SNe shows significant variety in the explosion parameters and in the characteristics of the host galaxies (although they seem to prefer moderately metal-poor environments). This would suggest for these objects a distribution in the space of parameters which is similar to that observed in more classical type IIP SNe, although they are intrinsically rare $(\lesssim 3 \%$ of all core-collapse $\mathrm{SNe}$ in a volume-limited sample).

Acknowledgements. This work is based in part on observations collected at the Italian 3.58-m Telescopio Nazionale Galileo (TNG), the 2.56-m Nordic Optical Telescope, the 2.2-m Telescope of the Centro Astronómico Hispano Alemàn (Calar Alto, Spain) and the 1.82-m Copernico Telescope on Cima Ekar (Asiago, Italy). The TNG is operated by the Fundación Galileo Galilei of the Instituto Nazionale di Astrofisica at the Spanish Observatorio del Roque de los Muchachos of the Instituto de Astrofisica de Canarias. We thank the support astronomers at the TNG, the 2.2-m Telescope at Calar Alto and the Nordic Optical Telescope for performing the follow-up observations of SN 2009E. S.B., F.B., E.C., M.T. and A.H. are partially supported by the PRIN-INAF 2009 with the project "Supernovae Variety and Nucleosynthesis Yields". M.L.P. acknowledges financial support from the Bonino-Pulejo Foundation. S.M. acknowledges financial support from the Academy of Finland (project: 8120503). We are grateful to the TriGrid VL project and the INAF-Astronomical Observatory of Padua for the use of computer facilities.

E.P. acknowledges the project Skylive of the Unione Astrofili Italiani (UAI) and the Skylive Remote Facility - Osservatorio B40 Skylive, Catania, Italy (http://www.skylive.it). We thank the amateur astronomers of the Associazione Astronomica Isaac Newton (http://www.isaacnewton.it) and of the Associazione Astronomica Cortina (http://www . cortinastelle. it/) for providing images of SN 2009E obtained during the SMMSS and CROSS supernova search programs: G. Iacopini (Osservatorio di Tavolaia, S. Maria a Monte, Pistoia), R. Mancini and F. Briganti (Stazione di Osservazione di Gavena, Cerreto Guidi, Italy), A. Dimai (Osservatorio di Col Driuscé, Cortina, Italy). We also thank J. M. Llapasset, O. Trondal, Boyd, for sharing their images with us, I. Bano and A. Englaro (Osservatorio Astronomico Polse di Cougnes) for their help with observations, A. Siviero for allowing ToO observations of SN 2009E at the 1.82-m Telescope of Asiago (Mt. Ekar, Italy) and T. Iijima for providing us the $\mathrm{B} \& \mathrm{C}$ spectrum obtained at the 1.22-m Galileo Telescope (Osservatorio Astrofisico di Asiago). A.P. is grateful to Mario Hamuy, Io Kleiser, Dovi Poznanski and Lisa Germany for sharing their data of SNe 2000cb and 1998bt.

This manuscript made wide use of information contained in the Bright Supernova web pages (maintained by D. Bishop), as part of the Rochester Academy of Sciences (http://www.RochesterAstronomy.org/ snimages/). We also used information collected from the the web site SNAude des Supernovae (http://www.astrosurf.com/snaude/). The paper is also based in part on data collected at the Akeno $50 \mathrm{~cm}$ Telescope (Akeno Observatory/ICRR, Yamanashi, Japan) and obtained from the SMOKA, which is operated by the Astronomy Data Center, National Astronomical
Observatory of Japan; and at the Faulkes North Telescope and obtained through the Faulkes Telescopes Data Archive.

\section{References}

Aikman, C., \& Newton, J. 1982, IAU Circ., 3690, 2

Aldering, G., \& Conley, A. 2000, IAU Circ., 7410, 3

Alexeyev, E. N., \& Alexeyeva, L. N. 2008, AstL, 34, 745

Arcavi, I., Gal-Yam, A., Kiewe, M., et al. 2009, Am. Astron. Soc. Meet., 214, 604.01

Armstrong, M. 2004, CBET, 82, 1

Arnett, W. D., Bahcall, J. N., Kirshner, R. P., \& Woosley, S. E. 1989, ARA\&A, 27, 629

Baba, H., Yasuda, N., Ichikawa, S., et al. 2002, Development of the SubaruMitaka-Okayama-Kiso Archive System, ADASS XI, ed. D. A. Bohlender, D. Durand, \& T. H. Handley, ASP Conf. Ser., 281, 298

Benetti, S., Turatto, M., Balberg, S., et al. 2001, MNRAS, 322, 361

Blanc, N., Copin, Y., Gangler, E., et al. 2006, Astron. Tel., 762, 1

Blondin, S., Modjaz, M., Kirshner, R., Challis, P., \& Calkins, M. 2006, CBET, 392,1

Blondin, S., Barbon, R., Pastorello, A., \& Calkins, M. 2008, CBET, 1300, 1

Bionta, R. M., Blewitt, G., Bratton, C. B., Caspere, D., \& Ciocio, A. 1987, Phys. Rew. Lett., 58, 1494

Boles, T. 2008, CBET, 1239, 1

Boles, T. 2009, CBET, 1648

Borkowski, K. J., Blondin, J. M., \& McCray, R. 1997, ApJ, 477, 281

Bresolin, F. 2007, ApJ, 656, 186

Cardelli, J. A., Clayton, G. C., \& Mathis, J. S. 1989, ApJ, 345, 245

Catchpole, R. M., Menzies, J. W., Monk, A. S., et al. 1987, MNRAS, 229, 15

Catchpole, R. M., Whitelock, P. A., Feast, M. W., et al. 1988, MNRAS, 231, 75

Catchpole, R. M., Whitelock, P. A., Menzies, J. W., et al. 1989, MNRAS, 237, 55

Chen, Y.-T., Yang, M., \& Lin, C.-S. 2006, CBET, 390, 1

Chugai, N. N. 1994, ApJ, 428, L17

Elmhamdi, A., Danziger, I. J., Chugai, N. N., et al. 2003, MNRAS, 338, 939

Filippenko, A. V., Ganeshalingham, M., \& Swift, B. J. 2004, IAU Circ., 8411, 1

Gal Yam, A., Cenko, S. B., Fox, D. B., et al. 2007, AIPC, 924, 297

Gilmozzi, R., Cassatella, A., Clavel, J., et al. 1987, Nature, 328, 318

Germany, L. 1998, IAU Circ., 6898

Germany, L. M., Reiss, D. J., Schmidt, B. P., Stubbs, C. W., \& Suntzeff, N. B. 2004, A\&A, 415, 863

Hakobyan, A. A., Mamom, G. A., Petrosian, A. R., Kunth, D., \& Turatto, M. 2009, A\&A, 508, 1259

Hamuy, M. 2001, Ph.D. Thesis, Univ. of Arizona

Hamuy, M., \& Suntzeff, N. B. 1990, AJ, 99, 1146

Hamuy, M., Suntzeff, N. B., Gonzales, R., \& Martin, G. 1988, AJ, 95, 63

Hirata, K., Kajita, T., Koshiba, M., Nakahata, M., \& Oyama, Y. 1987, Phys. Rew. Lett., 58, 1490

Inserra, C., Turatto. M., Pastorello, A., et al. 2011, MNRAS, 417, 261

Jarrett, T. H., Chester, T., Cutri, R., Schneider, S. E., \& Huchra, J. P. 2003, AJ, 125,525

Jha, S., Challis, P., \& Kirshner, R. 2000, IAU Circ. 7410, 2

Jones, D. H., Read, M. A., \& Saunders, W. 2009, MNRAS, 399, 683

Kennicutt, R. C. Jr., Bresolin, F., \& Garnett, D. R. 2003, ApJ, 591, 801

Kewley, L. J., Jansen, R. A., \& Geller, M. J. 2005, PASP, 117, 227

Kleiser, I. K. W., Poznanski, D., Kasen, D., et al. 2011, MNRAS, 415, 372

Kozma, C., \& Fransson, C. 1998, ApJ, 497, 431

Landolt, A. U. 1992, AJ, 104, 340

Li, H., \& McCray, R. 1992, ApJ, 387, 309

Li, W., Leaman, J., Chornock, R., et al. 2011, MNRAS, 412, 1441

Madison, D., \& Li, W. 2005, IAU Circ., 8541, 1

Madison, D., Li, W., \& Filippenko, A. 2008, CBET, 1239, 2

Maguire, K., di Carlo, E., Smartt, S. J., et al. 2010, MNRAS, 404, 981

Mazzali, P. A., \& Chugai, N. N. 1995, A\&A, 303, 118

Mazzali, P. A., Lucy, L. B., \& Butler, K. 1992, A\&A, 258, 399

Menzies, J. W., Catchpole, R. M., van Vuuren, G., et al. 1987, MNRAS, 227, 39

Modjaz, M., Kirshner, R., \& Challis, P. 2005, IAU Circ., 8542, 2

Navasardyan, H., Benetti, S., Bufano, F., \& Pastorello, A. 2009, CBET, 1738, 1

Papenkova, M., \& Li, W. 2000, IAU Circ., 7410, 1

Pastorello, A., Zampieri, L., Turatto, M., et al. 2004, MNRAS, 347, 74

Pastorello, A., Baron, E., Branch, D., et al. 2005, MNRAS, 360, 950

Pastorello, A., Sauer, D., Taubenberger, S., et al. 2006, MNRAS, 370, 1752

Pastorello, A., Quimby, R. M., Smartt, S. J., et al. 2008, MNRAS, 389, 131

Pastorello, A., Valenti, S., Zampieri, L., et al. 2009, MNRAS, 394, 2266

Patat, F., Barbon, R., Cappellaro, E., \& Turatto, M. 1994, A\&A, 282, 731

Phillips, M. M., Heathcote, S. R., Hamuy, M., \& Navarrete, M. 1988, AJ, 95, 1087 
Phillips, M. M., Hamuy, M., Heathcote, S. R., Suntzeff, N. B., \& Kirhakos, S. 1990, AJ, 99, 1133

Pilyugin, L. S., \& Thuan, T. X. 2007, ApJ, 669, 299

Pilyugin, L. S., Vílchez, J. M., \& Contini, T. 2004, A\&A, 425, 849

Poznanski, D., Ganeshalingam, M., Silverman, J. M., \& Filippenko, A. V. 2011, MNRAS, 415, L81

Pumo, M. L., Zampieri L., \& Turatto M., 2010, Mem. S. A. It. Suppl., 14, 123

Pumo, M. L., \& Zampieri L. 2011, ApJ, 741, 41

Prosperi, E., \& Hurst, G. H. 2009, CBET, 1734

Pun, C. S. J., Kirshner, R. P., Sonneborn, G., et al. 1995, ApJS, 99, 223

Rizzi, L., Tully, R. B., Makarov, D., et al. 2007, 661, 815

Saha, A., Thim, F., Tammann, G. A., Reindl, B., \& Sandage, A. 2006, ApJS, 165,108

Sakai, S., Ferrarese, L., Kennicutt, R. C., \& Saha, A. 2004, ApJ, 608, 42

Sandage, A., \& Tammann, G. A., 1974, ApJ, 194, 223

Schlegel, D. J., Finkbeiner, D. P., \& Davis, M. 1998, ApJ, 500, 525

Schmitz, M. F., \& Gaskell, C. M. 1988, in Supernova 1987A in the Large Magellanic Cloud, ed. M. Kafatos, \& A. Michalitsianos (Cambridge: Cambridge University Press), 112

Smartt, S. J., Eldridge, J. J., Crockett, R. M., \& Maund, J. R. 2009, MNRAS, 395,1409

Sonneborn, G., Altner, B., \& Kirshner, R. P. 1987, ApJ, 323, L35

Taddia, F., Stritzinger, M.D., Sollerman, J., et al. 2011, A\&A, in press [arXiv: 1111.2509]

Thielemann, F.-K., Nomoto, K., \& Hashimoto, M.-A., 1996, ApJ, 460, 408

Tsujimoto, T., \& Shigeyama, T. 2001, ApJ, 561, L97

Trondal, O., Luckas, P., \& Schwartz, M. 2006, CBET, 426, 1

Tsvetkov, D. Y. 1984, Astr. Tsirk., 1346, 1

Tsvetkov, D. Y. 1988, PZ, 22, 653

Tsvetkov, D. Y. 2008, PZ, 28, 3

Tsvetkov, D. Yu., Volnova, A. A., Shulga, A. P., et al. 2006, A\&A, 460, 769

Turatto, M., Mazzali, P. A., Young, T. R., et al. 1998, ApJ, 498, 129

Turatto, M., Cappellaro, E., \& Benetti, S. 2003, in From Twilight to Highlight: the Physics of Supernovae, ed. B. Leibundgut, W. Hillebrandt, Proc. to the ESO/MPA/MPE Workshop (an ESO Astrophysics Symp.) (Berlin: SpringerVerlag), 200

Udalski, A. 2003, Acta Astron., 53, 291

Udalski, A., Szymański, M. K., Soszyński, I., \& Poleski, R. 2008, Acta Astron., 58,69

Uomoto, A. 1986, ApJ, 310, L35

Utrobin, V. P., \& Chugai, N. N. 2005, A\&A, 441, 271

Utrobin, V. P., \& Chugai, N. N. 2011, A\&A, 532, A100

van den Bergh, S., \& McClure, R. D. 1989, ApJ, 347, L29

Whitelock, P. A., Catchpole, R. M., Menzies, J. W., et al. 1988, MNRAS, 234, 5

Whitelock, P. A., Catchpole, R. M., Menzies, J. W., et al., 1989, MNRAS, 240, 7

Williams, R. E. 1987, ApJ, 320, L117

Williams, A., Woodings, S., Martin, R., Verveer, A., \& Biggs, J. 1998, IAU Circ. 6805,2

Woodings, S. J., Williams, A. J., Martin, R., Burman, R. R., \& Blair, D. G. 1998, MNRAS, 301, L5

Woosley, S. E. 1988, ApJ, 330, 218

Woosley, S. E., \& Weaver, T. A. 1995, ApJS, 101, 181

Yamagata, T., \& Iye, M. 1982, IBVS, 2204, 1

Young, T. R., \& Branch, D. 1988, Nature, 333, 305
Young, D. R., Smartt, S. J., Valenti, S., et al. 2010, A\&A, 512, A70

Zampieri, L., Pastorello, A., Turatto, M., et al. 2003, MNRAS, 338, 711

Zaritsky, D., Harris, J., Thompson, I. B., \& Grebel, E. K. 2004, AJ, 128, 1606

1 INAF - Osservatorio Astronomico di Padova, Vicolo dell' Osservatorio 5, 35122 Padova, Italy

e-mail: andrea.pastorello@oapd.inaf.it

2 Astrophysics Research Centre, School of Mathematics and Physics, Queen's University Belfast, Belfast BT7 1NN, UK

3 Dipartimento di Astronomia, Università di Padova, Vicolo dell'Osservatorio 3, 35122 Padova, Italy

4 INAF - Osservatorio Astrofisico di Catania, via S. Sofia 78, 95123 Catania, Italy

5 INAF - Osservatorio Astronomico di Trieste, via G. B. Tiepolo 11, 34143 Trieste, Italy

6 Oskar Klein Centre, Department of Astronomy, AlbaNova, Stockholm University, 10691 Stockholm, Sweden

7 Tuorla Observatory, Department of Physics \& Astronomy, University of Turku, Väisäläntie 20, 21500 Piikkiö, Finland

8 Les Mauruches Observatoire, 364 Chemin de Notre Dame, 06220 Vallauris, France

9 Osservatorio Astronomico di Castelmartini, IAU 160, via Bartolini 1317, 51036 Larciano, Pistoia, Italy

10 Observatorio El Gujio, Onice 21, 28260 Galapagar, Madrid, Spain

11 Max-Planck-Institut für Astrophysik, Karl-Schwarzschild-Str. 1, 85741 Garching bei München, Germany

12 Coddenham Astronomical Observatory, Suffolk, UK

13 Osservatorio Astronomico di Tavolaia, Piazza della Vittoria 41, 56020 Santa Maria a Monte, Pisa, Italy

14 Private address, 105 Glen Pine Trail, Dawnsonville, GA 30543, USA

15 Osservatorio Astronomico Polse di Cougnes, Zuglio, 33020 Udine, Italy

16 Center for Astrophysics and Supercomputing, Swinburne University of Technology, Hawthorn, VIC 3122, Australia

17 Fundación Galileo Galilei - INAF, Telescopio Nazionale Galileo, 38700 Santa Cruz de la Palma, Tenerife, Spain

18 Private address, 1401 South A, Arkansas City, KS 67005, USA

19 The Astronomer, 16 Westminster Close, Basingstoke, Hants, RG22 4PP, UK

20 European Southern Observatory, Karl-Schwarzschild-Str. 2, 85748 Garching bei München, Germany

21 Dark Cosmology Centre, Niels Bohr Institute, University of Copenhagen, Juliane Maries Vej 30, 2100 Copenhagen, Denmark

22 Department of Physics and Astronomy, Western Kentucky University, 1906 College Heights Blvd., Bowling Green, KY 421011077, USA

23 University of Oklahoma, Health Science Center, 1100 N. Lindsay, Oklahoma City, OK 73104, USA

Pages 13 to 16 are available in the electronic edition of the journal at http://wWw . aanda.org 


\section{Appendix A: Basic information on the sample of SN 1987-like transients}

1. SN 1987A is the prototype of this family of H-rich corecollapse SNe, and -since it exploded in the nearby LMC- it is the best studied SN ever. The SN was discovered on 1987 February 24th by Shelton. Strong constraints on the time of the core-collapse came from the detection of a neutrino burst on February 23.316 UT by IMB and Kamiokande II (Bionta et al. 1987; Hirata et al. 1987). Extensive data sets at all wavelengths are provided by a large number of publications (e.g. Menzies et al. 1987; Catchpole et al. 1987, 1988, 1989; Whitelock et al. 1988, 1989; Hamuy et al. 1988; Phillips et al. 1988, 1990; Pun et al. 1995). More recent papers have unveiled the complex structure of the circumstellar environment of SN 1987A and its interaction with the SN ejecta (e.g. Borkowski et al. 1997). SN 1987A is one of the few objects for which we have robust contraints on the nature of the progenitor star. Pre-explosion images of the source at the SN position ( $\mathrm{Sk}-69202$ ) showed it to be a blue (B3 I) supergiant (Gilmozzi et al. 1987; Sonneborn et al. 1987) whose mass is estimated to be around $20 M_{\odot}$ (see Arnett et al. 1989, for a review). A small initial radius of the progenitor star is also used to explain the unusual, slow rise to maximum of the light curves of SN 1987A. In this paper we adopt as distance modulus of LMC and as total reddening toward SN 1987A the values of $\mu=18.50$ mag (Sakai et al. 2004) and $E(B-V)=0.19$ mag (Arnett et al. 1989).

2. SN 1909A is a historical SN that exploded in M101. Its photometric evolution shows striking similarity with that of SN 1987A (Young \& Branch 1988; Patat et al. 1994, see also Appendix B). A well-sampled light curve from photographic plates rescaled to the $B$-band system was published by Sandage \& Tammann (1974). We assume negligible extinction toward SN 1909A and a distance modulus of $\mu=29.34 \mathrm{mag}$ (Rizzi et al. 2007, see Table 4 for details). Although multiband observations are missing for this event, the $B$-band absolute magnitude at maximum $\left(M_{B} \approx-15.9\right.$, see Table 5) suggests that SN 1909A is one of the intrinsically brightest $\mathrm{SNe}$ in our sample.

3. SN $1982 F$ in NGC 4490 is a poorly followed under-luminous type II SN. Sparse data around maximum from photographic plates were published by Yamagata \& Iye (1982) and Tsvetkov (1984, 1988). The light curves share remarkable similarity with SN 2000cb (see below), more than with SN 1987A. No spectrum exists for this object to our knowledge.

4. SN 1998A is a well studied 1987A-like event which exploded in a spiral arm of the SBc galaxy IC 2627. The SN was discovered on 1998 January 6 by the Automated Supernova Search Program of the Perth Astronomy Research Group (PARG, Williams et al. 1998). Optical photometry and spectroscopy were published by Woodings et al. (1998) and Pastorello et al. (2005). Adopting the HyperLeda recessional velocity corrected for local infall onto Virgo $v_{\text {Vir }}$ $=1976 \mathrm{~km} \mathrm{~s}^{-1}$, we obtain a distance of $27.4 \mathrm{Mpc}(\mu=$ $32.19 \mathrm{mag}$ ). Since there was no evidence of additional extinction in the host galaxy, we adopt the same total reddening estimate as in Pastorello et al. (2005), i.e. $E(B-V)=$ $0.12 \mathrm{mag}$.

5. SN 1998bt was discovered in the Abell cluster 1736 by the Mount Stromlo Abell cluster supernova search team on March 10, 1998 (Germany 1998). Initially, no background galaxy was seen to be associated with the SN. However, subsequent deep imaging of the SN field revealed a very faint parent galaxy of $R=23.4$ (Germany et al. 2004). Unfortunately, spectroscopic classification for this SN does not exist. However, the overall behaviour of the light curve (see Fig. B.1) is reminiscent of that of SN 1987A.

6. SN 2000cb in IC 1158 is one of the best followed 1987A-like events in our sample. Discovered on April 27.42000 using the $0.8-\mathrm{m}$ Katzman Automatic Imaging Telescope (KAIT, Papenkova \& Li 2000), it was classified as a young type II SN by Jha et al. (2000) and Aldering \& Conley (2000). Optical photometric and spectroscopic observations have been presented by Hamuy (2001) and Kleiser et al. (2011). The photometric evolution of SN 2000cb is different from that of SN 1987A. The light curve in the $B$ band shows a maximum at $\approx 40$ days past core-collapse, followed by a slow decline up to 60 days and a short pseudo-plateau. At about 90 days a steep post plateau decline to the radioactive tail is visible, as in normal type IIP SNe. The evolution in the VRI bands is somewhat different, showing broad light curve peaks at 60-80 days (depending on the band) after corecollapse (Hamuy 2001; Kleiser et al. 2011). The light curve peaks are significantly broader than those of SN 1987A. We adopted the observed parameters of SN $2000 \mathrm{cb}$ as presented in Hamuy (2001). The explosion epoch, the adopted distance and the total reddening are those of Hamuy (2001). Their values are listed in Tables 4 and 5.

7. OGLE-2003-NOOS-005 never had a SN designation. It was discovered by the Optical Gravitational Lensing Experiment (OGLE, Udalski 2003) collaboration at $\alpha=5^{\mathrm{h}} 55^{\mathrm{m}} 38^{\mathrm{s}} .07$ and $\delta=-68^{\circ} 55^{\prime} 47^{\prime \prime} .1$ (J2000.0). It exploded in a faint spiral galaxy labelled by the NED database as 2MASX J05553978-6855381, behind LMC, and falls in the OGLE field LMC198.6 9 (Udalski et al. 2008). The well-followed Iband light curve of OGLE-2003-NOOS-005 ${ }^{12}$ well matches that of SN 1987A. Unfortunately multiband observations for this SN do not exist. The redshift of the host galaxy was spectroscopically determined measuring the positions of a few selected spectral features of a host galaxy spectrum obtained on 2009 August 14 with NTT (equipped with EFOSC2 and grism 11; resolution $=22 \AA$ ). Despite the low signal-tonoise, we identified a few absorption features (Ca II H\&K, the $g$-band $\lambda 4200, \mathrm{Mg} \mathrm{Ib} 25173)$, and $\mathrm{H} \alpha$ in emission. In addition the emission line of [O II] $\lambda 3727$ was marginally detected. This allowed to fix the redshift at $z=0.0302$ $\pm 0.0008\left(v_{\mathrm{rec}} \approx 9151 \pm 236 \mathrm{~km} \mathrm{~s}^{-1}\right)$, which corresponds to a distance of about $127 \mathrm{Mpc}(\mu=35.52 \mathrm{mag})$. These values are remarkably similar to those reported by NED $\left(v_{\text {rec }} \approx 9176 \pm 45 \mathrm{~km} \mathrm{~s}^{-1}\right.$; Jones et al. 2009). and HyperLeda $\left(v_{\text {rec }} \approx 9177 \pm 60 \mathrm{~km} \mathrm{~s}^{-1}\right)$. The recessional velocity corrected for Local Group infall into the Virgo Cluster is slightly lower $\left(v_{\text {Vir }} \approx 8882 \mathrm{~km} \mathrm{~s}^{-1}\right)$. Adopting the Hubble Constant value of $H_{0}=72 \mathrm{~km} \mathrm{~s}^{-1} \mathrm{Mpc}^{-1}$, we obtain a distance of 123.4 Mpc, i.e. distance modulus $\mu=35.46$ mag. We also assume in our analysis a total reddening toward the transient of $E(B-V)=0.16 \pm 0.10 \mathrm{mag}$. This was determined including the contribution of the Galaxy $E_{\mathrm{MW}}(B-V)=0.075 \mathrm{mag}$ (Schlegel et al. 1998) and the reddening at the position of 2MASX J05553978-6855381 due to the intrinsic contribution of LMC that was computed using the maps of Zaritsky et al. (2004) $\left(E_{\mathrm{LMC}}(B-V)=0.087 \mathrm{mag}\right)$. The $I$-band light curve of this $\mathrm{SN}$ is shown in Appendix B.

12 Available via ftp through the OGLE-III (2003 season) web site http://ogle. astrouw . edu.pl/ogle3/ews/N00S/2003/noos. html 
8. SN 2004em was discovered by Armstrong (2004) on Sept. 14, 2004 when the SN was at mag $=17.5$. The transient, later classified as a young type II by Filippenko et al. (2004), was hosted in an Sc galaxy (IC 1303). The object was significantly reddened by Galactic dust $(E(B-V)=0.108$, from Schlegel et al. 1998). Preliminary information on the Caltech Core-Collapse Project light curve has been provided by Gal Yam et al. (2007).

9. SN 2005ci, a LOSS discovery (Madison \& Li. 2005), was classified as a type II SN by Modjaz et al. (2005) on the basis of the presence of P-Cygni Balmer lines. Its peculiar light curve was first noted by Arcavi et al. (2009), and some earlytime unfiltered photometry was provided by Kleiser et al. (2011), showing a clear light curve rise by about 2.5 mag in $~ 45$ days. Unfortunately, with the modest amount of data available so far, we cannot estimate the main SN parameters. The host galaxy, NGC 5682, is an Sb-type; the Galaxy reddening is modest, $E(B-V)=0.033$ mag (Schlegel et al. 1998), but the relatively red continuum shown by the spectrum presented by Kleiser et al. (2011, see their Fig. 12) suggests a non negligible host galaxy reddening. The narrow host galaxy $\mathrm{Na}$ ID feature is not clearly detected and the amount of internal reddening cannot be constrained.

10. $S N 2006 V$ was discovered in the course of the Taiwan Supernova Survey on Feb. 4, 2006 UT at a magnitude of about 18 (Chen et al. 2006). The object was classified as a type II SN after maximum by Blondin et al. (2006) and extensively followed by the Carnegie Supernova Project collaboration, who noted the slowly rising, 1987A-like light curve (Taddia et al. 2011). The galaxy hosting SN 2006V is UGC 6510, which is classified as a face-on spiral (SABc, HyperLeda source) suffering of small Galactic reddening $(E(B-V)=0.029 \mathrm{mag}$, Schlegel et al. 1998).
The lack of detection of narrow Na ID absorption at the redshift of UGC 6510, suggest no additional host galaxy extinction toward SN 2006V (Taddia et al. 2011). A comparison between the multi-band light curves of SNe 2006V and 1987A can be found in Fig. 3 of Taddia et al. (2011).

11. SN 2006au was a discovery of the Tenagra Observatory Supernova Search using the 0.35-m Tenagra telescope in Oslo (on Mar. 7.20 UT). At the discovery, the object had mag 17.2 (Trondal et al. 2006). SN 2006au was later classified by the Nearby Supernova Factory as a type II SN (Blanc et al. 2006). Again, a comprehensive study is presented in Taddia et al. (2011) and comparisons with the light curves of SN 1987A are shown in their Fig. 4. The host galaxy, UGC 11057, is a late spiral (possibly an Sc-type, according to HyperLeda) with rather large Galactic reddening, i.e. $E(B-V)=0.172 \mathrm{mag}$ (Schlegel et al. 1998). However, Taddia et al. (2011) found that SN 2006au suffered also of large extinction due to dust in the host galaxy, deriving a total reddening of $E(B-V)_{\text {tot }}=0.312 \mathrm{mag}$.

\section{Appendix B: Light curves of 1987A-like events}

In this section, the light curves of seven 1987A-like type II SNe are compared with those of the prototype SN 1987A (solid or dashed blue lines, see Figs. B.1 and B.2). The light curves of SN 1987A are arbitrarily shifted in magnitude to match the peak magnitudes of the other objects. In the comparison, we assumed the JDs listed in Table 4 as explosion epochs. For a few individual events (e.g. SNe 1982F or 2000cb) the 1987A-likeness is more evident in the red bands than in the blue ones. As mentioned in Sect. 4, data for SN 2004em have not been published yet. 

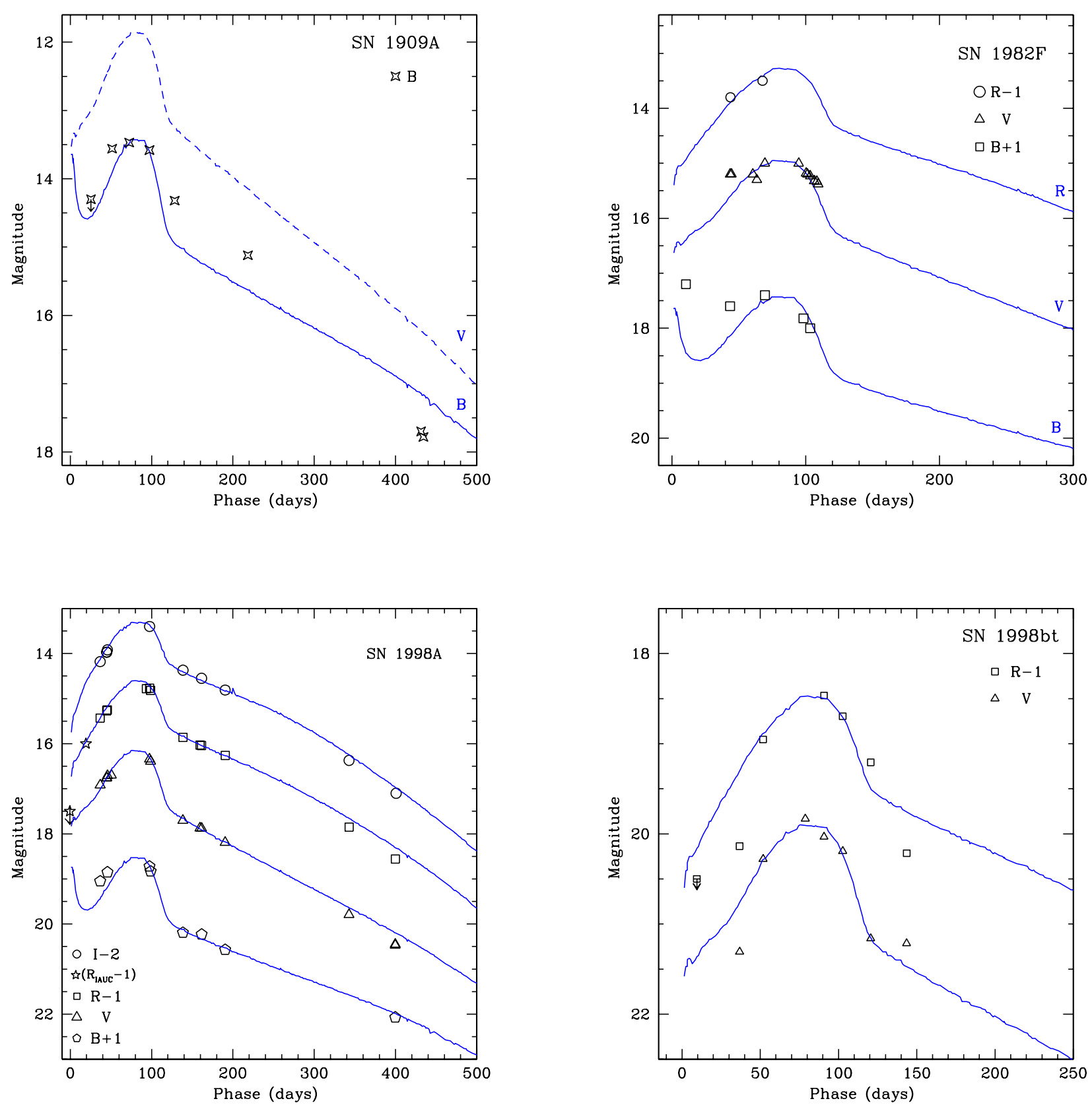

Fig. B.1. Light curves of 1987A-like events: first group of 4 events. The solid (or dashed) blue lines represent the light curves of SN 1987A, our template. The light curves of SN 1987A have been arbitrarily shifted in magnitude to match the peaks of the other transients. 

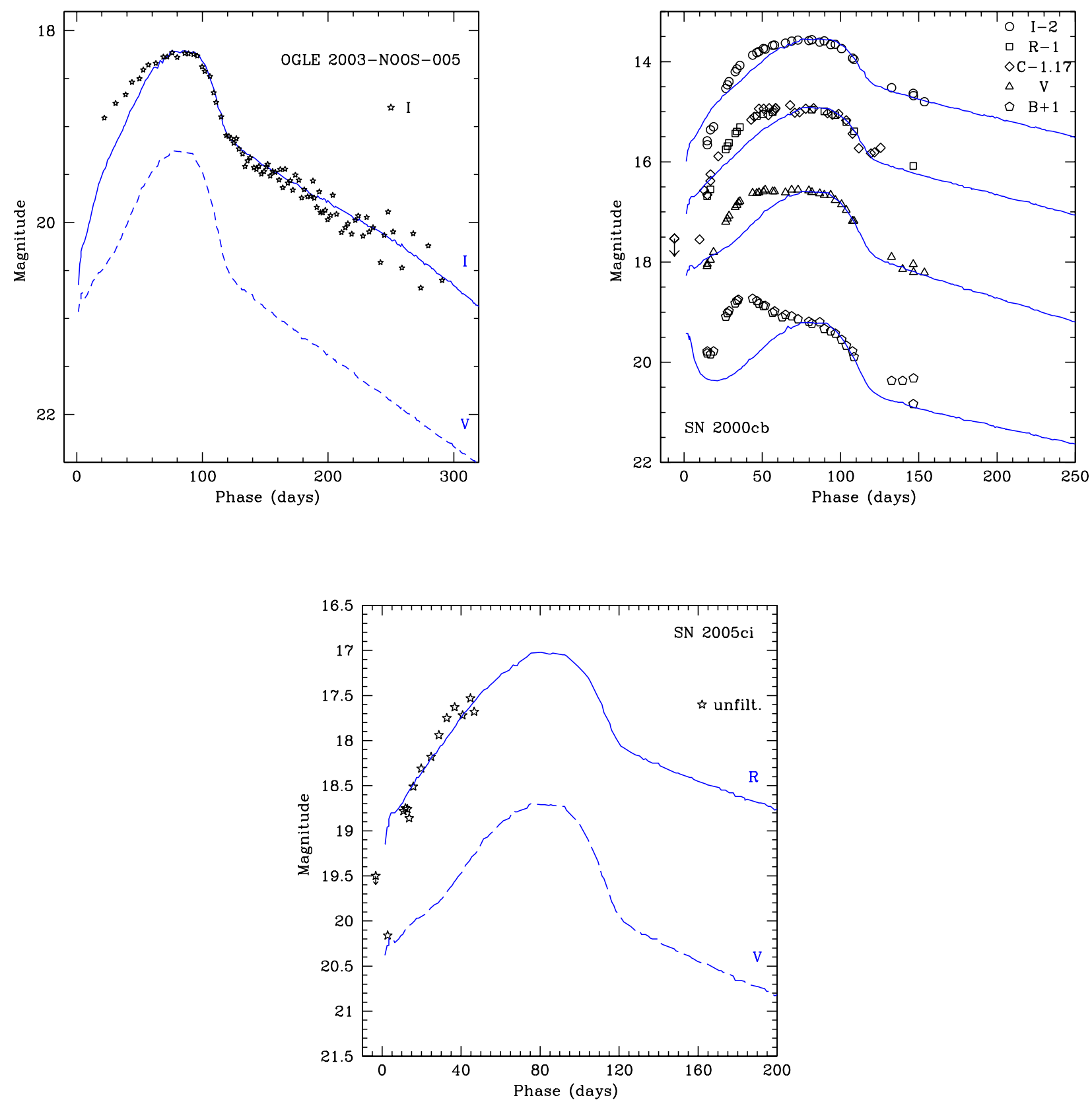

Fig. B.2. Light curves of 1987A-like events: second group of 3 events. The solid (and dashed) blue lines represent the light curves of SN 1987A, our template. The light curves of SN 1987A have been arbitrarily shifted in magnitude to match the peaks of the other transients. 